\title{
Distributional System Representations on Bandlimited Signals
}

\author{
Ullrich J. Mönich, Student Member, IEEE, and Holger Boche, Senior Member, IEEE
}

\begin{abstract}
In this paper we analyze the distributional convergence behavior of time-domain convolution type system representations on the Paley-Wiener space $\mathcal{P} \mathcal{W}_{\pi}^{1}$. Two convolution integrals as well as the discrete counterpart, the convolution sum, are treated. It is shown that there exist stable linear time-invariant (LTI) systems for which the convolution integral representation does not exist because the integral is divergent, even if the convergence is interpreted in a distributional sense. Furthermore, we completely characterize all stable LTI systems for which a convolution representation is possible by giving a necessary and sufficient condition for convergence. The classical and the distributional convergence behavior are compared, and differences between the convergence of the convolution integral and the convolution sum are discussed. Finally, the results are illustrated by numerical examples.
\end{abstract}

Index Terms-Bandlimited signal, convergence, convolution, distribution, system representation.

\section{INTRODUCTION}

$\mathbf{T}$ HE representation of bandlimited signals by sampling series or convolution integrals is important, not only from a theoretical point of view [1]-[3], but also for practical applications, where finite sums or integrals can be used for the signal approximation. Of course, the convergence behavior of both the sum and the integral is crucial. Oftentimes the interest is not in a series or convolution integral representation of the signal itself, but in a representation of some transformation of the signal, generated by some linear time-invariant (LTI) system [4], [5]. Then the goal is to find a system representation as a series or a convolution integral.

Many engineering books [6], [7] give the impression that any LTI system $T$ can be represented as a convolution integral in the form

$$
(T f)(t)=\int_{-\infty}^{\infty} f(\tau) h_{T}(t-\tau) \mathrm{d} \tau
$$

Manuscript received August 12, 2009; accepted May 24, 2010. Date of publication June 07, 2010; date of current version August 11, 2010. The associate editor coordinating the review of this manuscript and approving it for publication was Prof. Thierry Blu. The material in this paper was presented in part at the IEEE International Conference on Acoustics, Speech, and Signal Processing (ICASSP), Dallas, TX, 2010.

The authors are with the Technische Universität Berlin, Heinrich-Hertz-Chair for Mobile Communications, D-10578 Berlin, Germany (e-mail: ullrich. moenich@mk.tu-berlin.de; holger.boche@mk.tu-berlin.de).

Digital Object Identifier 10.1109/TSP.2010.2051804 where $h_{T}$ is the impulse response of the system and $f$ is the input signal. Of course this is true for example for stable LTI systems operating on bandlimited signals with finite energy. However, it is not necessarily true for stable LTI systems acting on other signal spaces. Note that the stability of the systems is defined always with respect to the norms of the considered signal spaces.

The problem of finding representations of stable LTI systems has been studied for a long time, and several results for spaces of bandlimited signals, which are larger than the space of bandlimited, finite energy signals, have been presented [5], [8]-[10]. In [5], Habib derived a convolution integral and a series representation for systems operating on bandlimited signals in the Zakai space [8], [11]. In [10], it has been shown that the integral in (1) is generally not convergent for signals from the Paley-Wiener space $\mathcal{P} \mathcal{W}_{\pi}^{1}$.

Although the integral in (1) does not necessarily exist in the classical sense for $\mathcal{P W}_{\pi}^{1}$, it might be possible that it can still be meaningfully interpreted in a distributional sense. Indeed, distributions can provide a way out of many convergence problems that are present in the classical nondistributional setting. One example is given by the convergence of Fourier series: It is well-known that there are signals in $L^{1}[-\pi, \pi]$ whose Fourier series diverge almost everywhere. In a distributional sense however, the Fourier series converges for all signals in $L^{1}[-\pi, \pi]$. This example shows that there are situation where a distributional interpretation can resolve convergence problems. Unfortunately, many engineering textbooks about LTI systems do not treat distributions in a rigorous mathematical manner. Often heuristic arguments prevail.

Another problem which has gained a lot of attention concerns the existence of the impulse response for stable LTI systems operating on general, not necessarily bandlimited, spaces, and the question whether the impulse response gives a complete description of the system [12]-[15]. In [12], it was shown that the class of stable (with respect to the $L^{\infty}$-norm) LTI systems that map bounded uniformly continuous signals into bounded uniformly continuous signals contains systems, whose impulse response is the zero function, but which take certain inputs into nonzero outputs. Consequently, there exist two different stable LTI systems that have the same impulse response. Reference [13] treats systems operating on bounded signals and finds a necessary and sufficient under which a systems has the representation (1).

The fact that the impulse response $h_{T}$ may not exist is one reason why a representation of the form (1) can be problematic. In [14] and [15], LTI systems were studied in a distributional way. The authors proved that in a distributional setting 
and under certain assumptions, it is possible to define in a certain sense an impulse response for every stable LTI system. One assumption that was made in order to obtain their results was that the space of input signals contains the space of test functions $\mathcal{D}$. Since functions in $\mathcal{D}$ are compactly supported, they cannot be bandlimited. Therefore, the results are not applicable for systems operating on spaces of bandlimited signals.

Fortunately, we do not have to face these problems here: Since we consider bandlimited input signals, the impulse response is always a well-defined bandlimited function, which uniquely determines the system. However, although the impulse response exists, it will turn out that stable LTI systems can generally not be represented in the form (1) because the integral diverges. In contrast to the common perception, this divergence cannot be circumvented by considering a distributional setting.

In this paper we analyze the distributional convergence behavior of the two convolution integrals

$$
\left(A_{N}^{T} f\right)(t):=\int_{-N}^{N} f(\tau) h_{T}(t-\tau) \mathrm{d} \tau
$$

and

$$
\left(B_{N}^{T} f\right)(t):=\int_{-N}^{N} f(t-\tau) h_{T}(\tau) \mathrm{d} \tau,
$$

and the convolution sum

$$
\left(S_{N}^{T} f\right)(t):=\sum_{k=-N}^{N} f(k) h_{T}(t-k)
$$

for signals $f$ in the Paley-Wiener space $\mathcal{P} \mathcal{W}_{\pi}^{1}$ and stable LTI systems $T$. The signal space $\mathcal{P} \mathcal{W}_{\pi}^{1}$ is the largest space in the scale of Paley-Wiener spaces. Thus, the results that are obtained for this space can be seen as an extension of the results for the well-known space $\mathcal{P} \mathcal{W}_{\pi}^{2}$ of bandlimited signals with finite energy. Furthermore, the space $\mathcal{P} \mathcal{W}_{\pi}^{1}$ is important because the convergence behavior of sampling series and convolution integrals for this space is closely related to the mean-square convergence behavior for wide-sense stationary stochastic processes [16]. We show that the perception that any stable LTI system acting on bandlimited signals can - at least in a distributional setting - be represented as a convolution integral is problematic and not justified in general. Moreover, we completely characterize all stable LTI systems for which the approximation processes (2), (3), and (4) converge to $T f$ for all $f \in \mathcal{P} \mathcal{W}_{\pi}^{1}$ as $N$ tends to infinity, and compare the distributional convergence behavior and the classical convergence behavior.

For practical applications we need the convergence of an approximation process for all signals from the signal space because generally it is not known in advance which signal from the signal space occurs in the application at hand. This is the reason why we want to characterize the stable LTI systems $T$ for which the approximation processes (2), (3), and (4) converge for all $f \in \mathcal{P} \mathcal{W}_{\pi}^{1}$.

The paper is organized as follows: In Section II we give some definitions and briefly review basic properties of stable LTI systems. Section III introduces distributions and the convergence of distributions. The main results about the distributional convergence behavior of the convolution integrals (2) and (3) are given in Section IV, whereas the discrete counterpart, the convolution sum (4), is treated in Section V. In Section VI some interesting differences between the convolution integral and the convolution sum are presented. Finally, the results are numerically illustrated in Section VII.

\section{NOTATION AND DEFINITIONS}

In order to continue, we need some notation and definitions. Let $\hat{f}$ denote the Fourier transform of a function $f$, where $\hat{f}$ is to be understood in the distributional sense. $L^{p}(\mathbb{R}), 1 \leq p<$ $\infty$, is the space of all $p$ th-power Lebesgue integrable functions on $\mathbb{R}$, with the usual norm $\|\cdot\|_{p}$, and $L^{\infty}(\mathbb{R})$ is the space of all functions for which the essential supremum norm $\|\cdot\|_{\infty}$ is finite. By $C[a, b], a, b \in \mathbb{R}$, we denote the set of all continuous functions on the interval $[a, b]$.

For $\sigma>0$ and $1 \leq p \leq \infty$ we denote by $\mathcal{P W}_{\sigma}^{p}$ the Paley-Wiener space of signals $f$ with a representation $f(z)=$ $1 /(2 \pi) \int_{-\sigma}^{\sigma} g(\omega) \mathrm{e}^{i z \omega} \mathrm{d} \omega, z \in \mathbb{C}$, for some $g \in L^{p}[-\sigma, \sigma]$. If $f \in \mathcal{P} \mathcal{W}_{\sigma}^{p}$ then $g(\omega)=\hat{f}(\omega)$. The norm for $\mathcal{P} \mathcal{W}_{\sigma}^{p}, 1 \leq p<\infty$, is given by $\|f\|_{\mathcal{P} \mathcal{W}_{\sigma}^{p}}=\left(1 /(2 \pi) \int_{-\sigma}^{\sigma}|\hat{f}(\omega)|^{p} \mathrm{~d} \omega\right)^{1 / p}$. For $p=2$ we obtain the Paley-Wiener space $\mathcal{P} \mathcal{W}_{\sigma}^{2}$, which is nothing else than the space of bandlimited signals with finite energy. By Hölder's inequality we have $\mathcal{P} \mathcal{W}_{\sigma}^{2} \subset \mathcal{P} \mathcal{W}_{\sigma}^{1}$. Moreover, it holds $\|f\|_{\infty} \leq\|f\|_{\mathcal{P} \mathcal{W}_{\sigma}^{p}}$, which implies that every signal in $\mathcal{P} \mathcal{W}_{\sigma}^{p}$, $1 \leq p \leq \infty$, is bounded on the real line.

We briefly review some facts about stable LTI systems, which will be needed afterward. A linear system $T: \mathcal{P} \mathcal{W}_{\pi}^{p} \rightarrow \mathcal{P} \mathcal{W}_{\pi}^{p}$, $1 \leq p \leq 2$, is called stable if the operator $T$ is bounded, i.e., if $\|T\|=\sup _{\|f\|_{\mathcal{P} \mathcal{W}_{\pi}^{p} \leq 1}}\|T f\|_{\mathcal{P} \mathcal{W}_{\pi}^{p}}<\infty$. Furthermore, it is called time-invariant if $(T f(\cdot-a))(t)=(T f)(t-a)$ for all $f \in \mathcal{P} \mathcal{W}_{\pi}^{p}$ and $t, a \in \mathbb{R}$.

Remark 1: Note that our definition of stability is with respect to the $\mathcal{P} \mathcal{W}_{\pi}^{p}$-norm and thus is different from the concept of bounded input-bounded output (BIBO) stability in general.

For every stable LTI system $T: \mathcal{P} \mathcal{W}_{\pi}^{1} \rightarrow \mathcal{P} \mathcal{W}_{\pi}^{1}$ there exists exactly one function $\hat{h}_{T} \in L^{\infty}[-\pi, \pi]$ such that

$$
(T f)(t)=\frac{1}{2 \pi} \int_{-\pi}^{\pi} \hat{f}(\omega) \hat{h}_{T}(\omega) \mathrm{e}^{i \omega t} \mathrm{~d} \omega
$$

for all $f \in \mathcal{P} \mathcal{W}_{\pi}^{1}$. The operator norm of $T$ is given by $\|T\|=$ $\left\|\hat{h}_{T}\right\|_{\infty}$ and the impulse response $h_{T}$ by $h_{T}=T$ sinc, where

$$
\operatorname{sinc}(t)= \begin{cases}\frac{\sin (\pi t)}{\pi t}, & t \neq 0 \\ 1, & t=0 .\end{cases}
$$

Conversely, every function $\hat{h}_{T} \in L^{\infty}[-\pi, \pi]$ defines a stable LTI system $T: \mathcal{P} \mathcal{W}_{\pi}^{1} \rightarrow \mathcal{P} \mathcal{W}_{\pi}^{1}$. Thus, the space of all stable LTI systems defined on $\mathcal{P} \mathcal{W}_{\pi}^{1}$ is isometrically isomorphic to $L^{\infty}[-\pi, \pi]$. Furthermore, it can be shown that the representation (5) with a unique function $\hat{h}_{T} \in L^{\infty}[-\pi, \pi]$ is also valid for all stable LTI systems $T: \mathcal{P} \mathcal{W}_{\pi}^{2} \rightarrow \mathcal{P} \mathcal{W}_{\pi}^{2}$ and that all $\hat{h}_{T} \in$ $L^{\infty}[-\pi, \pi]$ define a stable LTI system $T: \mathcal{P} \mathcal{W}_{\pi}^{2} \rightarrow \mathcal{P} \mathcal{W}_{\pi}^{2}$. Consequently, we do not have to distinguish between stable LTI systems that map $\mathcal{P} \mathcal{W}_{\pi}^{1}$ into $\mathcal{P} \mathcal{W}_{\pi}^{1}$ and stable LTI systems that map $\mathcal{P} \mathcal{W}_{\pi}^{2}$ into $\mathcal{P} \mathcal{W}_{\pi}^{2}$, because both can be identified with 
$\hat{h}_{T} \in L^{\infty}[-\pi, \pi]$. Therefore, every stable LTI system that maps $\mathcal{P W}_{\pi}^{1}$ in $\mathcal{P} \mathcal{W}_{\pi}^{1}$ maps $\mathcal{P} \mathcal{W}_{\pi}^{2}$ in $\mathcal{P} \mathcal{W}_{\pi}^{2}$, and vice versa. Note that $\hat{h}_{T} \in L^{\infty}[-\pi, \pi] \subset L^{2}[-\pi, \pi]$ and consequently $h_{T} \in \mathcal{P} \mathcal{W}_{\pi}^{2}$.

Thus, every stable LTI system on $\mathcal{P} \mathcal{W}_{\pi}^{1}$ has a frequency-domain representation according to (5). We will see that a time-domain representation in the form (1) is not always possible, even in a distributional setting.

\section{DistRIBUtions AND CONVERGENCE}

In order to be able to state our key results, we additionally need the concept of distributions. Distributions are continuous linear functionals on some space of test functions. In this paper we deal with two different test functions spaces. The first one is the space $\mathcal{D}$ of all functions $\phi: \mathbb{R} \rightarrow \mathbb{C}$ that have continuous derivatives of all orders and are zero outside some finite interval. $\mathcal{D}^{\prime}$ denotes the dual space of $\mathcal{D}$, i.e., the space of all distributions that can be defined on $\mathcal{D}$. The other space of test functions that we use in this paper is the Schwartz space $\mathcal{S}$ of all continuous functions $\phi: \mathbb{R} \rightarrow \mathbb{C}$ that have continuous derivatives of all orders and fulfill $\sup _{t \in \mathbb{R}}\left|t^{a} \phi^{(b)}(t)\right|<\infty$ for all $a, b \in \mathbb{N}_{0}=\mathbb{N} \cup\{0\} . \mathcal{S}^{\prime}$ denotes the dual space of $\mathcal{S}$. From the definition of the spaces $\mathcal{D}$ and $\mathcal{S}$, it follows immediately that $\mathcal{D}$ is a proper subspace of $\mathcal{S}$, and that $\mathcal{S}^{\prime}$ is a proper subspace of $\mathcal{D}^{\prime}$. Furthermore, we have $\|\phi\|_{\infty}<\infty$ and $\|\phi\|_{1}<\infty$ for all $\phi \in \mathcal{S}$, and consequently for all $\phi \in \mathcal{D}$. The Fourier transform maps the space $\mathcal{S}$ onto itself. These properties of $\phi$ will be used extensively in the proofs.

For a locally integrable functions $g$ we can define the linear functional

$$
\phi \mapsto \int_{-\infty}^{\infty} g(t) \phi(t) \mathrm{d} t
$$

on the space $\mathcal{D}$. It can be proven that this functional is continuous and thus defines a distribution [17]. If $g$ further fulfills $\int_{-\infty}^{\infty}|g(t)|(1+|t|)^{-m} \mathrm{~d} t<\infty$ for some $m \geq 0$ then (6) defines also a continuous linear functional on $\mathcal{S}$. Distributions of the type (6) are called regular distributions.

A sequence of distributions $\left\{f_{k}\right\}_{k \in \mathbb{N}}$ in $\mathcal{D}^{\prime}$ is said to converge in $\mathcal{D}^{\prime}$ if for every $\phi \in \mathcal{D}$ the sequence of numbers $\left\{f_{k} \phi\right\}_{k \in \mathbb{N}}$ converges. Equally, a sequence of distributions $\left\{f_{k}\right\}_{k \in \mathbb{N}}$ in $\mathcal{S}^{\prime}$ is said to converge in $\mathcal{S}^{\prime}$ if for every $\phi \in \mathcal{S}$ the sequence of numbers $\left\{f_{k} \phi\right\}_{k \in \mathbb{N}}$ converges. Thus, a sequence of regular distributions, which is induced by a sequence of functions $\left\{g_{k}\right\}_{k \in \mathbb{N}}$ according to (6), converges in $\mathcal{S}^{\prime}$ if for every $\phi \in \mathcal{S}$ the sequence of numbers $\left\{\int_{-\infty}^{\infty} g_{k}(t) \phi(t) \mathrm{d} t\right\}_{k \in \mathbb{N}}$ converges.

Convergence in $\mathcal{S}^{\prime}$ and convergence in $\mathcal{D}^{\prime}$ are connected in the following way.

Observation 1: If $\left\{f_{k}\right\}_{k \in \mathbb{Z}}$ is a sequence in $\mathcal{S}^{\prime}$ it is also a sequence in $\mathcal{D}^{\prime}$, and, since $\mathcal{D} \subset \mathcal{S}$, convergence in $\mathcal{S}^{\prime}$ implies convergence in $\mathcal{D}^{\prime}$.

For further details about distributions, and for a definition of convergence in the test spaces, we would like to refer the reader to [17].

\section{CONVOLUTiOn InTEGRAL}

In this section, we analyze the convergence behavior of the two convolution integrals (2) and (3) for stable LTI systems $T$. Note that, for all $N \in \mathbb{N}, A_{N}^{T} f$ and $B_{N}^{T} f$ are bounded and continuous functions and therefore can be identified with a regular distribution according to (6).

The theory for stable LTI systems operating on bandlimited signals with finite energy is simple. It is well known that every stable LTI system $T: \mathcal{P} \mathcal{W}_{\pi}^{2} \rightarrow \mathcal{P} \mathcal{W}_{\pi}^{2}$ has the representation

$$
(T f)(t)=\int_{-\infty}^{\infty} f(t-\tau) h_{T}(\tau) \mathrm{d} \tau=\int_{-\infty}^{\infty} f(\tau) h_{T}(t-\tau) \mathrm{d} \tau
$$

with $h_{T}=$ Tsinc.

However, the situation for signals $f \in \mathcal{P} \mathcal{W}_{\pi}^{1}$ is more difficult. In [10] it has been shown that the convolution integrals (2) and (3) have a significantly different convergence behavior. For example, it has been shown for the Hilbert transform that (3) is globally uniformly convergent for all $f \in \mathcal{P} \mathcal{W}_{\pi}^{1}$, but that there are signals in $\mathcal{P} \mathcal{W}_{\pi}^{1}$ for which the peak value of (2) diverges. Further, the class of systems for which (2) and (3) converge pointwise has been completely characterized. It turned out that there are stable LTI systems for which the integrals (2) and (3) diverge pointwise. More precisely, for every $t \in \mathbb{R}$ there is a stable LTI systems $T$ such that (2) diverges for some signal $f \in \mathcal{P} \mathcal{W}_{\pi}^{1}$ as $N$ tends to infinity. The same is true for the convolution integral (3).

Although the convolution integrals are not necessarily convergent in the classical (pointwise) sense, it may be possible that (2) and (3), interpreted as a sequence of regular distributions, converge in the distributional sense for all stable LTI systems $T$ and all $f \in \mathcal{P} \mathcal{W}_{\pi}^{1}$. If this was true the common conception that every stable LTI system has time-domain representation in the form of a convolution integral would get a rigorous theoretical foundation for the space $\mathcal{P} \mathcal{W}_{\pi}^{1}$, at least in a distributional sense.

In this section we analyze this question and show that there are stable LTI systems and signals in $\mathcal{P} \mathcal{W}_{\pi}^{1}$ for which (2) and (3) diverge even in the distributional sense. Furthermore, we completely characterize all stable LTI systems for which we have convergence in the distributional sense by giving a necessary and sufficient condition for convergence. By characterizing the distributional convergence behavior we extend results from [10].

\section{A. Convergence Behavior of the Convolution Integral I}

We start our analysis with the convergence behavior of the convolution integral (2). For notational convenience, we introduce the abbreviation

$$
A_{N, \phi}^{T} f:=\int_{-\infty}^{\infty}\left(A_{N}^{T} f\right)(t) \phi(t) \mathrm{d} t .
$$

In the following theorem we completely characterize the stable LTI systems for which $\left(A_{N}^{T} f\right)(t)$ converges in the classical (pointwise) sense to $(T f)(t)$ for all $f \in \mathcal{P} \mathcal{W}_{\pi}^{1}$. Moreover, we characterize the stable LTI systems for which $A_{N}^{T} f$ converges in the distributional sense to $T f$ for all $f \in \mathcal{P} \mathcal{W}_{\pi}^{1}$. 
Theorem 1: Let $T: \mathcal{P} \mathcal{W}_{\pi}^{1} \rightarrow \mathcal{P} \mathcal{W}_{\pi}^{1}$ be a stable LTI system. i) For all $t \in \mathbb{R}$ and all $f \in \mathcal{P} \mathcal{W}_{\pi}^{1}$ we have

$$
\lim _{N \rightarrow \infty}\left|(T f)(t)-\left(A_{N}^{T} f\right)(t)\right|=0
$$

if and only if there exists a constant $C_{1}<\infty$ such that

$$
\max _{\omega \in[-\pi, \pi]}\left|\frac{1}{\pi} \int_{-\pi}^{\pi} \hat{h}_{T}\left(\omega_{1}\right) \frac{\sin \left(N\left(\omega-\omega_{1}\right)\right)}{\omega-\omega_{1}} \mathrm{~d} \omega_{1}\right| \leq C_{1}
$$

for all $N \in \mathbb{N}$. In addition, if (8) is not fulfilled, then for every $t \in \mathbb{R}$ there exists a signal $f_{1} \in \mathcal{P} \mathcal{W}_{\pi}^{1}$ such that

$$
\limsup _{N \rightarrow \infty}\left|\left(A_{N}^{T} f_{1}\right)(t)\right|=\infty .
$$

ii) Moreover, we have

$$
\lim _{N \rightarrow \infty}\left|A_{N, \phi}^{T} f-\int_{-\infty}^{\infty}(T f)(t) \phi(t) \mathrm{d} t\right|=0
$$

for all $f \in \mathcal{P} \mathcal{W}_{\pi}^{1}$ and all $\phi \in \mathcal{S}$ if and only if for all $\phi \in \mathcal{S}$ there exists a constant $C_{2}=C_{2}(\phi)<\infty$ such that

$\max _{\omega \in[-\pi, \pi]}\left|\frac{1}{\pi} \int_{-\pi}^{\pi} \hat{h}_{T}\left(\omega_{1}\right) \hat{\phi}\left(-\omega_{1}\right) \frac{\sin \left(N\left(\omega-\omega_{1}\right)\right)}{\omega-\omega_{1}} \mathrm{~d} \omega_{1}\right| \leq C_{2}(\phi)$

for all $N \in \mathbb{N}$. In addition, if (11) is not fulfilled for some $\phi \in \mathcal{S}$, then there exists a signal $f_{1} \in \mathcal{P} \mathcal{W}_{\pi}^{1}$ such that

$$
\limsup _{N \rightarrow \infty}\left|A_{N, \phi}^{T} f_{1}\right|=\infty \text {. }
$$

Since (8) does not depend on $t$, we have the special situation that the convergence of $\left(A_{N}^{T} f\right)(t)$ for some $t \in \mathbb{R}$ and all $f \in \mathcal{P} \mathcal{W}_{\pi}^{1}$ implies the convergence of $\left(A_{N}^{T} f\right)(t)$ for all $t \in \mathbb{R}$ and all $f \in \mathcal{P W}_{\pi}^{1}$. Due to this special behavior we are able to derive the interesting result in Theorem 2 that pointwise convergence for some $t \in \mathbb{R}$ and all $f \in \mathcal{P W}_{\pi}^{1}$ is equivalent to distributional convergence for all $f \in \mathcal{P} \mathcal{W}_{\pi}^{1}$. Moreover, we will see in Section V that the convolution sum does not possess this behavior.

In addition to the pointwise convergence behavior, Theorem 1 characterizes the convergence of $A_{N}^{T} f$ in $\mathcal{S}^{\prime} . A_{N}^{T} f$ converges to $T f$ in $\mathcal{S}^{\prime}$ for all $f \in \mathcal{P} \mathcal{W}_{\pi}^{1}$ if and only if for all $\phi \in \mathcal{S}$ there exists a constant $C_{2}(\phi)$ such that (11) is fulfilled for all $N \in \mathbb{N}$. Moreover, if (11) is not fulfilled for some $\phi \in \mathcal{S}$ then we have distributional divergence of $A_{N}^{T} f_{1}$ for some $f_{1} \in \mathcal{P} \mathcal{W}_{\pi}^{1}$ in the sense of (12).

Note that $\int_{-\infty}^{\infty}(T f)(t) \phi(t) \mathrm{d} t$ is always some finite number, because $T f \in \mathcal{P} \mathcal{W}_{\pi}^{1}$ is bounded. For this reason (12) implies that

$$
\limsup _{N \rightarrow \infty}\left|A_{N, \phi}^{T} f_{1}-\int_{-\infty}^{\infty}\left(T f_{1}\right)(t) \phi(t) \mathrm{d} t\right|=\infty
$$

For the proof of Theorem 1 we need Lemma 1, which is proved in Appendix B.

Lemma 1: For all stable LTI systems $T: \mathcal{P} \mathcal{W}_{\pi}^{1} \rightarrow \mathcal{P} \mathcal{W}_{\pi}^{1}$, $\phi \in \mathcal{S}$, and $N \in \mathbb{N}$ we have

$$
\begin{aligned}
& \sup _{\|f\|_{\mathcal{P} \mathcal{W}_{\pi}^{1}} \leq 1}\left|A_{N, \phi}^{T} f\right| \\
& \quad=\max _{\omega \in[-\pi, \pi]}\left|\frac{1}{\pi} \int_{-\pi}^{\pi} \hat{h}_{T}\left(\omega_{1}\right) \hat{\phi}\left(-\omega_{1}\right) \frac{\sin \left(N\left(\omega-\omega_{1}\right)\right)}{\omega-\omega_{1}} \mathrm{~d} \omega_{1}\right| .
\end{aligned}
$$

Proof of Theorem 1: Part i) was already proved in [10]. It remains to prove ii). First, we prove the " $\Leftarrow$ " direction of the "if and only if" statement, and second, the " $\Rightarrow$ " direction as well as (12).

" $\Leftarrow$ " direction: Let $f \in \mathcal{P} \mathcal{W}_{\pi}^{1}, \phi \in \mathcal{S}$, and $\epsilon>0$ be arbitrary but fixed. Since $\mathcal{P} \mathcal{W}_{\pi}^{2}$ is dense in $\mathcal{P} \mathcal{W}_{\pi}^{1}$, there exists a function $f_{\epsilon} \in \mathcal{P} \mathcal{W}_{\pi}^{2}$ with $\left\|f-f_{\epsilon}\right\|_{\mathcal{P} \mathcal{W}_{\pi}^{1}}<\epsilon$. According to Lemma 1 and the assumption (11) we have

$$
\begin{aligned}
& A_{N, \phi}^{T}\left(f-f_{\epsilon}\right) \leq\left\|f-f_{\epsilon}\right\|_{\mathcal{P} \mathcal{W}_{\pi}^{1}} \\
& \times \max _{\omega \in[-\pi, \pi]}\left|\frac{1}{\pi} \int_{-\pi}^{\pi} \hat{h}_{T}\left(\omega_{1}\right) \hat{\phi}\left(-\omega_{1}\right) \frac{\sin \left(N\left(\omega-\omega_{1}\right)\right)}{\omega-\omega_{1}} \mathrm{~d} \omega_{1}\right| \leq \epsilon C_{2}(\phi)
\end{aligned}
$$

for all $N \in \mathbb{N}$. Therefore, we obtain

$$
\begin{aligned}
& \left|\int_{-\infty}^{\infty}(T f)(t) \phi(t) \mathrm{d} t-A_{N, \phi}^{T} f\right| \\
& =\mid \int_{-\infty}^{\infty}\left(T\left(f-f_{\epsilon}\right)\right)(t) \phi(t) \mathrm{d} t \\
& \quad+\int_{-\infty}^{\infty}\left(T f_{\epsilon}\right)(t) \phi(t) \mathrm{d} t-A_{N, \phi}^{T} f_{\epsilon}-A_{N, \phi}^{T}\left(f-f_{\epsilon}\right) \mid \\
& <\left\|T\left|\left\||| f-f_{\epsilon}\right\|_{\mathcal{P} \mathcal{W}_{\pi}^{1}} \int_{-\infty}^{\infty}\right| \phi(t) \mid \mathrm{d} t\right. \\
& \quad+\left|\int_{-\infty}^{\infty}\left(T f_{\epsilon}\right)(t) \phi(t) \mathrm{d} t-A_{N, \phi}^{T} f_{\epsilon}\right|+\epsilon C_{2}(\phi) \\
& <\epsilon|| T \mid\|\phi\|_{1} \\
& \quad+\left|\int_{-\infty}^{\infty}\left(T f_{\epsilon}\right)(t) \phi(t) \mathrm{d} t-A_{N, \phi}^{T} f_{\epsilon}\right|+\epsilon C_{2}(\phi) .
\end{aligned}
$$

Further, we have

$$
\begin{aligned}
A_{N, \phi}^{T} f_{\epsilon} & =\int_{-\infty}^{\infty}\left(A_{N}^{T} f_{\epsilon}\right)(t) \phi(t) \mathrm{d} t \\
& =\int_{-\infty}^{\infty} \int_{-N}^{N} f_{\epsilon}(t) h_{T}(t-\tau) \mathrm{d} \tau \phi(t) \mathrm{d} t .
\end{aligned}
$$


Since $f_{\epsilon}, h_{T} \in \mathcal{P} \mathcal{W}_{\pi}^{2}$,

$$
\begin{aligned}
\left|\int_{-N}^{N} f_{\epsilon}(t) h_{T}(t-\tau) \mathrm{d} \tau \phi(t)\right| & \leq \int_{-\infty}^{\infty}\left|f_{\epsilon}(t) h_{T}(t-\tau)\right| \mathrm{d} \tau|\phi(t)| \\
& \leq\left\|f_{\epsilon}\right\|_{2}\left\|h_{T}\right\|_{2}|\phi(t)|,
\end{aligned}
$$

and $\phi \in L^{1}(\mathbb{R})$, we can apply Lebesgue's dominated convergence theorem, which leads to

$$
\begin{aligned}
\lim _{N \rightarrow \infty} A_{N, \phi}^{T} f_{\epsilon} & =\int_{-\infty}^{\infty} \int_{-\infty}^{\infty} f_{\epsilon}(t) h_{T}(t-\tau) \mathrm{d} \tau \phi(t) \mathrm{d} t \\
& =\int_{-\infty}^{\infty}\left(T f_{\epsilon}\right)(t) \phi(t) \mathrm{d} t
\end{aligned}
$$

where the last equality follows from

$$
\int_{-\infty}^{\infty} f_{\epsilon}(\tau) h_{T}(t-\tau) \mathrm{d} \tau=\frac{1}{2 \pi} \int_{-\pi}^{\pi} \hat{f}_{\epsilon}(\omega) \hat{h}_{T}(\omega) \mathrm{e}^{i \omega t}=\left(T f_{\epsilon}\right)(t)
$$

according to the generalized Parseval equality. Thus, there is a $N_{0}=N_{0}(\epsilon)$ such that

$$
\left|\int_{-\infty}^{\infty}\left(T f_{\epsilon}\right)(t) \phi(t) \mathrm{d} t-A_{N, \phi}^{T} f_{\epsilon}\right|<\epsilon
$$

for all $N \geq N_{0}(\epsilon)$. Combining (13) and (14), we obtain

$$
\left|\int_{-\infty}^{\infty}(T f)(t) \phi(t) \mathrm{d} t-A_{N, \phi}^{T} f\right|<\epsilon\left(\|T\|\|\mid \phi\|_{1}+C_{2}(\phi)+1\right)
$$

for all $N \geq N_{0}(\epsilon)$. This completes this part of the proof, because $\epsilon>0$ was arbitrary.

“ $\Rightarrow$ " direction: Let $\phi \in \mathcal{S}$ be arbitrary but fixed. Since

$$
\left|A_{N, \phi}^{T} f\right| \leq\left|A_{N, \phi}^{T} f-\int_{-\infty}^{\infty}(T f)(t) \phi(t) \mathrm{d} t\right|+\left|\int_{-\infty}^{\infty}(T f)(t) \phi(t) \mathrm{d} t\right|
$$

for all $N \in \mathbb{N}$ and all $f \in \mathcal{P} \mathcal{W}_{\pi}^{1}$, (10) and the fact that

$$
\left|\int_{-\infty}^{\infty}(T f)(t) \phi(t) \mathrm{d} t\right| \leq\left\|T \left|\|\mid f\|_{\mathcal{P} \mathcal{W}_{\pi}^{1}}\|\phi\|_{1}<\infty\right.\right.
$$

imply that $\sup _{N \in \mathbb{N}}\left|A_{N, \phi}^{T} f\right|<\infty$ for all $f \in \mathcal{P} \mathcal{W}_{\pi}^{1}$. Since $A_{N, \phi}^{T}: \mathcal{P} \mathcal{W}_{\pi}^{1} \rightarrow \mathbb{C}$ is a bounded linear operator for all $N \in \mathbb{N}$, it follows from the Banach-Steinhaus theorem [18, p. 98] that

$$
\sup _{N \in \mathbb{N}\|f\|_{\mathcal{P W}}^{1} \leq 1} \sup _{\pi, \phi}\left|A_{N}^{T} f\right|<\infty .
$$

Consequently, we have

$$
\sup _{N \in \mathbb{N}} \max _{\omega \in[-\pi, \pi]}\left|\frac{1}{\pi} \int_{-\pi}^{\pi} \hat{h}_{T}\left(\omega_{1}\right) \hat{\phi}\left(-\omega_{1}\right) \frac{\sin \left(N\left(\omega-\omega_{1}\right)\right)}{\omega-\omega_{1}} \mathrm{~d} \omega_{1}\right|<\infty
$$

by Lemma 1, which completes this part of the "if and only if" statement.

On the other hand, if (11) is not fulfilled, i.e., if $\limsup _{N \rightarrow \infty} \max _{\omega \in[-\pi, \pi]} \mid \frac{1}{\pi} \int_{-\pi}^{\pi} \hat{h}_{T}\left(\omega_{1}\right) \hat{\phi}\left(-\omega_{1}\right) \times$

$$
\frac{\sin \left(N\left(\omega-\omega_{1}\right)\right)}{\omega-\omega_{1}} \mathrm{~d} \omega_{1} \mid=\infty
$$

we have $\sup _{N \in \mathbb{N}} \sup _{\|f\|_{\mathcal{P W} 1} \leq 1}\left|A_{N, \phi}^{T} f\right|=\infty$ according to Lemma 1. Thus, the Banach-Steinhaus theorem [18, p. 98] implies that there exists a signal $f_{1} \in \mathcal{P} \mathcal{W}_{\pi}^{1}$ such that (12) is true.

Remark 2: In the previous theorem we have seen that if (11) is not fulfilled then there exists a signal $f_{1} \in \mathcal{P} \mathcal{W}_{\pi}^{1}$ such that $A_{N}^{T} f_{1}$ diverges in $\mathcal{S}^{\prime}$. In this case, there is not only one single divergence creating signal. In fact, the set of signals for which we have divergence is large: It is a residual set, i.e., the complement of a set of the first category, and therefore it is dense in $\mathcal{P W}_{\pi}^{1}[19$, p. 12].

From the proof of part ii) of Theorem 1 we see that the same arguments hold if we replace $\phi \in \mathcal{S}$ with $\phi \in \mathcal{D}$. Thus, part ii) of Theorem 1 is also true if we replace $\phi \in \mathcal{S}$ with $\phi \in \mathcal{D}$. With that we also have a characterization of the convergence of $A_{N}^{T} f$ in $\mathcal{D}^{\prime}$.

Corollary 1: Part ii) of Theorem 1 remains true if $\phi \in \mathcal{S}$ is replaced with $\phi \in \mathcal{D}$.

In Theorem 3 we will show that there really exists a stable LTI system such that (11) is not fulfilled for some $\phi \in \mathcal{D}$, i.e., that there exists a stable LTI system $T_{1}$ such that $A_{N}^{T_{1}} f_{1}$ diverges in $\mathcal{D}^{\prime}$ for some $f_{1} \in \mathcal{P} \mathcal{W}_{\pi}^{1}$.

It would be interesting to have a connection between the pointwise convergence of $A_{N}^{T} f$, the convergence of $A_{N}^{T} f$ in $\mathcal{D}^{\prime}$, and the convergence of $A_{N}^{T} f$ in $\mathcal{S}^{\prime}$. The following lemma, the proof of which is given in Appendix $\mathrm{C}$, is the main step towards Theorem 2, where we identify this connection.

Lemma 2: Let $T: \mathcal{P} \mathcal{W}_{\pi}^{1} \rightarrow \mathcal{P} \mathcal{W}_{\pi}^{1}$ be a stable LTI system.

i) If for all $\phi \in \mathcal{D}$ there exists a constant $C_{2}=C_{2}(\phi)<\infty$ such that (11) in Theorem 1 is fulfilled for all $N \in \mathbb{N}$ then there exists a constant $C_{1}<\infty$ such that (8) in Theorem 1 is fulfilled for all $N \in \mathbb{N}$.

ii) Further, if there exists a constant $C_{1}<\infty$ such that (8) in Theorem 1 is fulfilled for all $N \in \mathbb{N}$ then, for all $\phi \in \mathcal{S}$ there exists a constant $C_{2}=C_{2}(\phi)<\infty$ such that (11) in Theorem 1 is fulfilled for all $N \in \mathbb{N}$.

Theorem 2 establishes the connection between the classical (pointwise) convergence and the distributional convergence of $A_{N}^{T} f$.

Theorem 2: Let $T: \mathcal{P} \mathcal{W}_{\pi}^{1} \rightarrow \mathcal{P} \mathcal{W}_{\pi}^{1}$ be a stable LTI system. The following statements are equivalent.

i) $A_{N}^{T} f$ converges in $\mathcal{D}^{\prime}$ for all $f \in \mathcal{P} \mathcal{W}_{\pi}^{1}$.

ii) $A_{N}^{T} f$ converges in $\mathcal{S}^{\prime}$ for all $f \in \mathcal{P} \mathcal{W}_{\pi}^{1}$.

iii) $\left(A_{N}^{T} f\right)(t)$ converges pointwise for some $t \in \mathbb{R}$ and all $f \in \mathcal{P} \mathcal{W}_{\pi}^{1}$.

iv) $\left(A_{N}^{T} f\right)(t)$ converges pointwise for all $t \in \mathbb{R}$ and all $f \in$ $\mathcal{P W}_{\pi}^{1}$.

Proof: "iii) $\Rightarrow$ ii)": This follows from Theorem 1 i), Lemma 2 ii), and Theorem 1 ii). "ii) $\Rightarrow$ i)": Observation 1. 
“i) $\Rightarrow i v) ":$ This follows from Corollary 1, Lemma 2 i), and Theorem 1 i). "iv) $\Rightarrow$ iii)": Obvious.

In general, convergence in $\mathcal{S}^{\prime}$ is a stronger statement than convergence in $\mathcal{D}^{\prime}$, because the former implies the latter. However, in Theorem 2 we have the situation that $A_{N}^{T} f$ converges in $\mathcal{S}^{\prime}$ if and only if it converges in $\mathcal{D}^{\prime}$.

Moreover, Theorem 2 shows that we do not gain anything regarding the convergence behavior of $A_{N}^{T} f$ for stable LTI systems $T$ and signals $f$ in $\mathcal{P} \mathcal{W}_{\pi}^{1}$ if we consider the more relaxed concept of distributional convergence. If $\left(A_{N}^{T} f\right)(t)$ diverges in the classical (pointwise) sense for some signal $f \in \mathcal{P} \mathcal{W}_{\pi}^{1}$ and some $t \in \mathbb{R}$ then $A_{N}^{T} f$ diverges also in $\mathcal{D}^{\prime}$ and consequently in $\mathcal{S}^{\prime}$ for some signal $f \in \mathcal{P W}_{\pi}^{1}$.

The following theorem states that there exists a stable LTI system $T_{1}$ and a signal $f_{1} \in \mathcal{P} \mathcal{W}_{\pi}^{1}$ such that $A_{N}^{T_{1}} f_{1}$ diverges in $\mathcal{D}^{\prime}$ as $N$ tends to infinity.

Theorem 3: There exists a stable LTI system $T_{1}: \mathcal{P} \mathcal{W}_{\pi}^{1} \rightarrow$ $\mathcal{P} \mathcal{W}_{\pi}^{1}$ and a signal $f_{1} \in \mathcal{P} \mathcal{W}_{\pi}^{1}$ such that

$$
\limsup _{N \rightarrow \infty}\left|A_{N, \phi_{1}}^{T_{1}} f_{1}\right|=\infty
$$

for some $\phi_{1} \in \mathcal{D}$.

Proof: We can prove this theorem by finding an explicit system $T_{1}$ such that

$$
\limsup _{N \rightarrow \infty} \max _{\omega \in[-\pi, \pi]}\left|\frac{1}{\pi} \int_{-\pi}^{\pi} \hat{h}_{T_{1}}\left(\omega_{1}\right) \frac{\sin \left(N\left(\omega-\omega_{1}\right)\right)}{\omega-\omega_{1}} \mathrm{~d} \omega_{1}\right|=\infty .
$$

Then it follows by Lemma 2 i) that there exists a $\phi_{1} \in \mathcal{D}$ such that

$$
\begin{aligned}
\limsup _{N \rightarrow \infty} \max _{\omega \in[-\pi, \pi]} \mid \frac{1}{\pi} \int_{-\pi}^{\pi} \hat{h}_{T_{1}}\left(\omega_{1}\right) \hat{\phi}_{1}\left(-\omega_{1}\right) \times & \\
& \frac{\sin \left(N\left(\omega-\omega_{1}\right)\right)}{\omega-\omega_{1}} \mathrm{~d} \omega_{1} \mid=\infty
\end{aligned}
$$

which in turn implies, by Corollary 1 , that there exists a signal $f_{1} \in \mathcal{P} \mathcal{W}_{\pi}^{1}$ such that (15) is true.

Next, we construct the system $T_{1}$. To this end, consider

$$
h_{H}(t)=\frac{1}{2 \pi} \int_{-\pi}^{\pi}(-i \operatorname{sgn}(\omega)) \mathrm{e}^{i \omega t} \mathrm{~d} \omega=\frac{\sin ^{2}\left(\frac{\pi}{2} t\right)}{\frac{\pi}{2} t}
$$

and the function $h_{N}$, defined by $h_{N}(\tau)=\left(h_{H}(\tau+N)-h_{H}(\tau-\right.$ $N)) / 2$. It follows that

$$
\hat{h}_{N}(\omega)=\sin (N \omega) \operatorname{sgn}(\omega), \quad|\omega| \leq \pi
$$

and

$$
\begin{aligned}
\int_{-N}^{N} h_{N}(\tau) \mathrm{d} \tau & =\frac{1}{2 \pi} \int_{-\pi}^{\pi} \hat{h}_{N}(\omega) \int_{-N}^{N} \mathrm{e}^{i \omega \tau} \mathrm{d} \tau \mathrm{d} \omega \\
& =\frac{1}{\pi} \int_{-\pi}^{\pi} \sin (N \omega) \operatorname{sgn}(\omega) \frac{\sin (N \omega)}{\omega} \mathrm{d} \omega \\
& =\frac{2}{\pi} \int_{0}^{\pi} \frac{\sin ^{2}(N \omega)}{\omega} \mathrm{d} \omega>\frac{1}{\pi} \log (2 N)
\end{aligned}
$$

because

$$
\begin{aligned}
\frac{2}{\pi} \int_{0}^{\pi} \frac{\sin ^{2}(N \omega)}{\omega} \mathrm{d} \omega & =\int_{0}^{2 N} \frac{\sin ^{2}\left(\frac{\pi}{2} \tau\right)}{\frac{\pi}{2} \tau} \mathrm{d} \tau \\
& >\frac{2}{\pi} \sum_{k=0}^{2 N-1} \frac{1}{k+1} \int_{k}^{k+1} \sin ^{2}\left(\frac{\pi}{2} \tau\right) \mathrm{d} \tau \\
& =\frac{1}{\pi} \sum_{k=0}^{2 N-1} \frac{1}{k+1}>\frac{1}{\pi} \log (2 N) .
\end{aligned}
$$

Now let $N_{k}=2^{n_{k}}, k \in \mathbb{N}$, be a sequence of dyadic numbers, where $\left\{n_{k}\right\}_{k=1}^{\infty}$ is a sequence of natural numbers satisfying $n_{k+1}>n_{k}, k \in \mathbb{N}$, and let $\left\{\epsilon_{k}\right\}_{k=1}^{\infty}$ be a sequence of positive numbers with $\sum_{k=1}^{\infty} \epsilon_{k}<\infty$. Consider the function

$$
\hat{h}_{T_{1}}(\omega)=\sum_{k=1}^{\infty} \epsilon_{k} \hat{h}_{N_{k}}(\omega) \text {. }
$$

Since

$$
\left\|\hat{h}_{T_{1}}\right\|_{L^{\infty}[-\pi, \pi]} \leq \sum_{k=1}^{\infty} \epsilon_{k}\left\|\hat{h}_{N_{k}}\right\|_{L^{\infty}[-\pi, \pi]} \leq \sum_{k=1}^{\infty} \epsilon_{k}<\infty,
$$

the operator $T_{1}$ defined by

$$
\left(T_{1} f\right)(t)=\frac{1}{2 \pi} \int_{-\pi}^{\pi} \hat{f}(\omega) \hat{h}_{T_{1}}(\omega) \mathrm{e}^{i \omega t} \mathrm{~d} \omega
$$

is a stable LTI system, and the impulse response $h_{T_{1}}$ is a well defined continuous function.

Next, we analyze the integral $\int_{-M}^{M} h_{T_{1}}(\tau) \mathrm{d} \tau$ for arbitrary dyadic numbers $M \geq 2$. We have

$$
\begin{aligned}
\int_{-M}^{M} h_{T_{1}}(\tau) \mathrm{d} \tau & =\frac{1}{\pi} \int_{-\pi}^{\pi} \hat{h}_{T_{1}}(\omega) \frac{\sin (M \omega)}{\omega} \mathrm{d} \omega \\
& =\sum_{k=1}^{\infty} \epsilon_{k} \frac{1}{\pi} \int_{-\pi}^{\pi} \operatorname{sgn}(\omega) \sin \left(N_{k} \omega\right) \frac{\sin (M \omega)}{\omega} \mathrm{d} \omega \\
& =\sum_{k=1}^{\infty} \epsilon_{k} \frac{2}{\pi} \int_{0}^{\pi} \sin \left(N_{k} \omega\right) \frac{\sin (M \omega)}{\omega} \mathrm{d} \omega
\end{aligned}
$$


The right-hand side of (19) is further analyzed. For all $k \in \mathbb{N}$ and $M=N_{k}$ we have

$$
\frac{2}{\pi} \int_{0}^{\pi} \sin \left(N_{k} \omega\right) \frac{\sin (M \omega)}{\omega} \mathrm{d} \omega \geq \frac{1}{\pi} \log \left(2 N_{k}\right) .
$$

Furthermore, for every $k \in \mathbb{N}$ and $M \neq N_{k}$ we can decompose the integral into three parts by splitting the integration interval and using the identity for the product of sine functions

$$
\begin{aligned}
& \frac{2}{\pi} \int_{0}^{\pi} \sin \left(N_{k} \omega\right) \frac{\sin (M \omega)}{\omega} \mathrm{d} \omega=\frac{2}{\pi} \int_{0}^{\delta} \sin \left(N_{k} \omega\right) \frac{\sin (M \omega)}{\omega} \mathrm{d} \omega \\
& +\frac{1}{\pi} \int_{\delta}^{\pi} \frac{\cos \left(\left(M-N_{k}\right) \omega\right)}{\omega} \mathrm{d} \omega-\frac{1}{\pi} \int_{\delta}^{\pi} \frac{\cos \left(\left(M+N_{k}\right) \omega\right)}{\omega} \mathrm{d} \omega .
\end{aligned}
$$

By choosing $\bar{\delta}_{k, M}=\pi /\left(2\left|N_{k}-M\right|\right)$ it can be shown that, for all $k \in \mathbb{N}$ and $N_{k}, M$ dyadic with $N_{k} \neq M$,

$$
\begin{aligned}
& \left|\frac{2}{\pi} \int_{0}^{\bar{\delta}_{k, M}} \sin \left(N_{k} \omega\right) \frac{\sin (M \omega)}{\omega} \mathrm{d} \omega\right| \leq 1, \\
& \left|\frac{1}{\pi} \int_{\bar{\delta}_{k, M}}^{\pi} \frac{\cos \left(\left(M-N_{k}\right) \omega\right)}{\omega} \mathrm{d} \omega\right| \leq C_{3}
\end{aligned}
$$

and

$$
\left|\frac{1}{\pi} \int_{\bar{\delta}_{k, M}}^{\pi} \frac{\cos \left(\left(M+N_{k}\right) \omega\right)}{\omega} \mathrm{d} \omega\right| \leq C_{4} .
$$

Hence, for $N_{k}, M$ dyadic and $N_{k} \neq M$ we have

$$
\left|\frac{2}{\pi} \int_{0}^{\pi} \sin \left(N_{k} \omega\right) \frac{\sin (M \omega)}{\omega} \mathrm{d} \omega\right| \leq C_{5}
$$

for all $k \in \mathbb{N}$. By setting $M=N_{r}$, we obtain

$$
\left|\int_{-N_{r}}^{N_{r}} h_{T_{1}}(\tau) \mathrm{d} \tau\right| \geq \epsilon_{r} \frac{1}{\pi} \log \left(2 N_{r}\right)-C_{5} \sum_{\substack{k=1 \\ k \neq r}}^{\infty} \epsilon_{k} .
$$

The function $h_{T_{1}}$, which was defined in (18), certainly depends on the concrete choice of the sequences $\left\{\epsilon_{k}\right\}_{k=1}^{\infty}$ and $\left\{N_{k}\right\}_{k=1}^{\infty}$. We can choose $\epsilon_{k}=1 / k^{2}$ and $N_{k}=2^{\left(k^{3}\right)}$. Then the function $h_{T_{1}}$ satisfies

$$
\begin{aligned}
\left|\int_{-N_{r}}^{N_{r}} h_{T_{1}}(\tau) \mathrm{d} \tau\right| & \geq \frac{1}{\pi} \frac{1}{r^{2}} \log \left(2^{\left(r^{3}\right)}\right)-C_{5} \sum_{\substack{k=1 \\
k \neq r}}^{\infty} \frac{1}{k^{2}} \\
& \geq \frac{\log (2)}{\pi} r-C_{5} \frac{\pi^{2}}{6}
\end{aligned}
$$

and consequently

$$
\limsup _{N \rightarrow \infty}\left|\int_{-N}^{N} h_{T_{1}}(\tau) \mathrm{d} \tau\right|=\infty .
$$

Moreover, since

$$
\begin{aligned}
& \limsup _{N \rightarrow \infty}\left|\int_{-N}^{N} h_{T_{1}}(\tau) \mathrm{d} \tau\right| \\
& \quad \leq \limsup _{N \rightarrow \infty} \max _{\omega \in[-\pi, \pi]}\left|\int_{-N}^{N} \mathrm{e}^{-i \omega \tau} h_{T_{1}}(\tau) \mathrm{d} \tau\right| \\
& \quad=\limsup _{N \rightarrow \infty} \max _{\omega \in[-\pi, \pi]}\left|\frac{1}{\pi} \int_{-\pi}^{\pi} \hat{h}_{T_{1}}\left(\omega_{1}\right) \frac{\sin \left(N\left(\omega-\omega_{1}\right)\right)}{\omega-\omega_{1}} \mathrm{~d} \omega_{1}\right|,
\end{aligned}
$$

we have (16).

In Section VII-A we will use the system $T_{1}$ that was constructed in the proof of Theorem 3 to illustrate the divergence of (16) by a numerical example.

Theorem 3 shows that a convolution type representation of stable LTI systems in the form (2) is not possible in general for the space $\mathcal{P} \mathcal{W}_{\pi}^{1}$, even if the convergence is treated in the distributional sense. In Section IV-C we will see that the same is true for the second convolution integral (3).

\section{B. Test Signals}

Before we treat the second convolution integral, we give an interesting interpretation of condition (8) in terms of test signals. Since

$$
\begin{aligned}
\frac{1}{\pi} \int_{-\pi}^{\pi} \hat{h}_{T}\left(\omega_{1}\right) \frac{\sin \left(N\left(\omega-\omega_{1}\right)\right)}{\omega-\omega_{1}} \mathrm{~d} \omega_{1} & =\int_{-N}^{N} \mathrm{e}^{i \omega \tau} h_{T}(-\tau) \mathrm{d} \tau \\
& =\left(A_{N}^{T} \mathrm{e}^{i \omega \cdot}\right)(0)
\end{aligned}
$$

we see that (8) is equivalent to $\max _{\omega \in[-\pi, \pi]}\left|\left(A_{N}^{T} f_{\omega}^{\text {test }}\right)(0)\right| \leq$ $C_{1}$, where $f_{\omega}^{\text {test }}(t)=\mathrm{e}^{i \omega t}$. Thus, we can regard the exponential function $\mathrm{e}^{i \omega t}$ as a test signal. If $\left|\left(A_{N}^{T} f_{\omega}^{\text {test }}\right)(0)\right|$ is uniformly bounded for all test signals $f_{\omega}^{\text {test }}$, where the parameter $\omega$ ranges from $-\pi$ to $\pi$, and all $N \in \mathbb{N}$, then $A_{N}^{T} f$ converges pointwise, and due to Theorem 2 also in $\mathcal{S}^{\prime}$, for all $f \in \mathcal{P} \mathcal{W}_{\pi}^{1}$. That is, we have

$$
\lim _{N \rightarrow \infty}\left|A_{N, \phi}^{T} f-\int_{-\infty}^{\infty}(T f)(t) \phi(t) \mathrm{d} t\right|=0
$$

for all $f \in \mathcal{P} \mathcal{W}_{\pi}^{1}$ and all $\phi \in \mathcal{S}$.

However, the converse statement might be more practicable. If we find one test signal $f_{\omega_{1}}^{\text {test }}, \omega_{1} \in[-\pi, \pi]$, such that $\lim _{N \rightarrow \infty}\left|\left(A_{N}^{T} f_{\omega_{1}}^{\text {test }}\right)(0)\right|=\infty$ then we have both pointwise divergence and divergence in $\mathcal{D}^{\prime}$. That is, there exists a signal $f_{1} \in \mathcal{P} \mathcal{W}_{\pi}^{1}$ such that $\limsup _{N \rightarrow \infty}\left|A_{N, \phi_{1}}^{T} f_{1}\right|=\infty$ for some $\phi_{1} \in \mathcal{D}$. 
Note the simple structure of the test signals: They are just scaled versions of one basic function $\mathrm{e}^{i t}$. Obviously, this set of test signals is neither dense in $\mathcal{P W}_{\pi}^{1}$ nor does it form a linear space.

\section{Convergence Behavior of the Convolution Integral II}

Now we treat (3), i.e., the second convolution integral.

The next theorem analyzes the global convergence behavior of $B_{N}^{T}$ and the distributional convergence behavior of $B_{N}^{T}$ in $\mathcal{S}^{\prime}$. For each type of convergence, we completely characterize the stable LTI systems $T$ for which $B_{N}^{T} f$ converges to $T f$ for all $f \in \mathcal{P} \mathcal{W}_{\pi}^{1}$, by giving a necessary and sufficient condition for convergence.

Theorem 4: Let $T: \mathcal{P} \mathcal{W}_{\pi}^{1} \rightarrow \mathcal{P} \mathcal{W}_{\pi}^{1}$ be a stable LTI system.

i) For all $f \in \mathcal{P} \mathcal{W}_{\pi}^{1}$ we have

$$
\lim _{N \rightarrow \infty}\left\|T f-B_{N}^{T} f\right\|_{\infty}=0
$$

if and only if there exists a constant $C_{6}<\infty$ such that

$$
\max _{\omega \in[-\pi, \pi]}\left|\frac{1}{\pi} \int_{-\pi}^{\pi} \hat{h}_{T}\left(\omega_{1}\right) \frac{\sin \left(N\left(\omega-\omega_{1}\right)\right)}{\omega-\omega_{1}} \mathrm{~d} \omega_{1}\right| \leq C_{6}
$$

for all $N \in \mathbb{N}$. In addition, if (21) is not fulfilled, then there exists a signal $f_{1} \in \mathcal{P} \mathcal{W}_{\pi}^{1}$ such that

$$
\limsup _{N \rightarrow \infty}\left\|B_{N}^{T} f_{1}\right\|_{\infty}=\infty .
$$

ii) Moreover, we have

$$
\lim _{N \rightarrow \infty}\left|B_{N, \phi}^{T} f-\int_{-\infty}^{\infty}(T f)(t) \phi(t) \mathrm{d} t\right|=0
$$

for all $f \in \mathcal{P} \mathcal{W}_{\pi}^{1}$ and all $\phi \in \mathcal{S}$ if and only if for all $\phi \in \mathcal{S}$ there exists a constant $C_{7}=C_{7}(\phi)<\infty$ such that

$\max _{\omega \in[-\pi, \pi]}\left|\hat{\phi}(-\omega) \frac{1}{\pi} \int_{-\pi}^{\pi} \hat{h}_{T}\left(\omega_{1}\right) \frac{\sin \left(N\left(\omega-\omega_{1}\right)\right)}{\omega-\omega_{1}} \mathrm{~d} \omega_{1}\right| \leq C_{7}(\phi)$

for all $N \in \mathbb{N}$. In addition, if (23) is not fulfilled for some $\phi \in \mathcal{S}$, then there exists a signal $f_{1} \in \mathcal{P} \mathcal{W}_{\pi}^{1}$ such that

$$
\limsup _{N \rightarrow \infty}\left|B_{N, \phi}^{T} f_{1}\right|=\infty \text {. }
$$

The proof of Theorem 4 is done analogously to the proof of Theorem 1.

We see that the conditions (8) and (21) are the same. Therefore, $A_{N}^{T} f$ converges pointwise for all $f \in \mathcal{P} \mathcal{W}_{\pi}^{1}$ if and only if $B_{N}^{T} f$ converges uniformly for all $f \in \mathcal{P} \mathcal{W}_{\pi}^{1}$.

Moreover, Theorem 4 gives a necessary and sufficient condition for the convergence of $B_{N}^{T} f$ in $\mathcal{S}^{\prime} . B_{N}^{T} f$ converges to $T f$ in $\mathcal{S}^{\prime}$ for all $f \in \mathcal{P} \mathcal{W}_{\pi}^{1}$ if and only if for all $\phi \in \mathcal{S}$ there exists a constant $C_{7}(\phi)$ such that (23) is fulfilled for all $N \in \mathbb{N}$.

Since the proof of part ii) of Theorem 4 is analogously to the proof of Theorem 1, we here have the same situation and can replace $\phi \in \mathcal{S}$ with $\phi \in \mathcal{D}$. This observation leads to the next corollary about the convergence of $B_{N}^{T} f$ in $\mathcal{D}^{\prime}$.

Corollary 2: Part ii) of Theorem 4 remains true if $\phi \in \mathcal{S}$ is replaced with $\phi \in \mathcal{D}$.

Corollary 2 provides a necessary and sufficient condition for the convergence of $B_{N}^{T} f$ in $\mathcal{D}^{\prime}$. $B_{N}^{T} f$ converges to $T f$ in $\mathcal{D}^{\prime}$ for all $f \in \mathcal{P} \mathcal{W}_{\pi}^{1}$ if and only if for all $\phi \in \mathcal{D}$ there exists a constant $C_{7}(\phi)$ such that (23) is fulfilled for all $N \in \mathbb{N}$.

Of course, we are again interested in a connection between the uniform convergence of $B_{N}^{T} f$, the convergence of $B_{N}^{T} f$ in $\mathcal{D}^{\prime}$, and the convergence of $B_{N}^{T} f$ in $\mathcal{S}^{\prime}$. The following lemma, the proof of which is similar to the proof of Lemma 2, is the main step towards Theorem 5, where we identify this connection.

Lemma 3: Let $T: \mathcal{P} \mathcal{W}_{\pi}^{1} \rightarrow \mathcal{P} \mathcal{W}_{\pi}^{1}$ be a stable LTI system.

i) If for all $\phi \in \mathcal{D}$ there exists a constant $C_{7}=C_{7}(\phi)<\infty$ such that (23) in Theorem 4 is fulfilled for all $N \in \mathbb{N}$ then there exists a constant $C_{6}<\infty$ such that (21) in Theorem 4 is fulfilled for all $N \in \mathbb{N}$.

ii) Further, if there exists a constant $C_{6}<\infty$ such that (21) in Theorem 4 is fulfilled for all $N \in \mathbb{N}$ then, for all $\phi \in \mathcal{S}$, there exists a constant $C_{7}=C_{7}(\phi)<\infty$ such that (23) in Theorem 4 is fulfilled for all $N \in \mathbb{N}$.

Theorem 5 shows that again we do not have to distinguish between convergence in $\mathcal{D}^{\prime}$ and convergence in $\mathcal{S}^{\prime}$ because both are equivalent.

Theorem 5: Let $T: \mathcal{P} \mathcal{W}_{\pi}^{1} \rightarrow \mathcal{P} \mathcal{W}_{\pi}^{1}$ be a stable LTI system. The following statements are equivalent.

i) $B_{N}^{T} f$ converges in $\mathcal{D}^{\prime}$ for all $f \in \mathcal{P} \mathcal{W}_{\pi}^{1}$.

ii) $B_{N}^{T} f$ converges in $\mathcal{S}^{\prime}$ for all $f \in \mathcal{P} \mathcal{W}_{\pi}^{1^{*}}$.

iii) $B_{N}^{T} f$ converges uniformly on all of $\mathbb{R}$ for all $f \in \mathcal{P} \mathcal{W}_{\pi}^{1}$.

Proof: "iii) $\Rightarrow$ ii)": This follows from Theorem 4 i), Lemma 3 i), and Theorem 3 ii). "ii) $\Rightarrow i$ )": Observation 1. " $i) \Rightarrow$ iii)": This follows from Corollary 2, Lemma 3 i), and Theorem 4 i).

With Corollary 2 we have completely characterized all stable LTI systems $T$ for which $B_{N}^{T} f$ converges in $\mathcal{D}^{\prime}$ for all $f \in$ $\mathcal{P W}_{\pi}^{1}$. Next we show that there actually exists a stable LTI system $T_{1}$ such that $B_{N}^{T_{1}} f_{1}$ diverges in $\mathcal{D}^{\prime}$ for some $f_{1} \in \mathcal{P} \mathcal{W}_{\pi}^{1}$.

Theorem 6: There exists a stable LTI system $T_{1}: \mathcal{P} \mathcal{W}_{\pi}^{1} \rightarrow$ $\mathcal{P} \mathcal{W}_{\pi}^{1}$ and a signal $f_{1} \in \mathcal{P} \mathcal{W}_{\pi}^{1}$ such that

$$
\limsup _{N \rightarrow \infty}\left|B_{N, \phi_{1}}^{T_{1}} f_{1}\right|=\infty
$$

for some $\phi_{1} \in \mathcal{D}$.

Proof: In the proof of Theorem 3 we have constructed a stable LTI system $T_{1}$ such that

$$
\limsup _{N \rightarrow \infty} \max _{\omega \in[-\pi, \pi]}\left|\frac{1}{\pi} \int_{-\pi}^{\pi} \hat{h}_{T_{1}}\left(\omega_{1}\right) \frac{\sin \left(N\left(\omega-\omega_{1}\right)\right)}{\omega-\omega_{1}} \mathrm{~d} \omega_{1}\right|=\infty .
$$

It follows from Lemma 3 i) that there exists a $\phi_{1} \in \mathcal{D}$ such that $\limsup _{N \rightarrow \infty} \max _{\omega \in[-\pi, \pi]}\left|\hat{\phi}_{1}(-\omega) \frac{1}{\pi} \int_{-\pi}^{\pi} \hat{h}_{T_{1}}\left(\omega_{1}\right) \frac{\sin \left(N\left(\omega-\omega_{1}\right)\right)}{\omega-\omega_{1}} \mathrm{~d} \omega_{1}\right|$ which in turn implies, by Corollary 2, that there exists a signal $f_{1} \in \mathcal{P} \mathcal{W}_{\pi}^{1}$ for which (24) is true. 


\section{Comparison of the Convergence Behavior of the Convolution Integrals I and II}

In general, the convolution integrals (2) and (3) have a different convergence behavior. This can be seen for example, if the stable LTI system is the Hilbert transform $H: \mathcal{P W}_{\pi}^{1} \rightarrow$ $\mathcal{P} \mathcal{W}_{\pi}^{1}$, defined by

$$
(H f)(t)=\frac{1}{2 \pi} \int_{-\pi}^{\pi}(-i \operatorname{sgn}(\omega)) \hat{f}(\omega) \mathrm{e}^{i \omega t} \mathrm{~d} \omega .
$$

For the Hilbert transform, $B_{N}^{H} f$ is uniformly convergent for all $f \in \mathcal{P} \mathcal{W}_{\pi}^{1}$. In contrast, $A_{N}^{H} f$ is not uniformly convergent for all $f \in \mathcal{P} \mathcal{W}_{\pi}^{1}$, because the peak value of $A_{N}^{H} f_{1}$ diverges for some signal $f_{1} \in \mathcal{P} \mathcal{W}_{\pi}^{1}$.

Theorem 7: We have

$$
\lim _{N \rightarrow \infty}\left\|H f-B_{N}^{H} f\right\|_{\infty}=0
$$

for all $f \in \mathcal{P} \mathcal{W}_{\pi}^{1}$, but there exists a signal $f_{1} \in \mathcal{P} \mathcal{W}_{\pi}^{1}$ such that

$$
\limsup _{N \rightarrow \infty}\left\|H f-A_{N}^{H} f\right\|_{\infty}=\infty .
$$

For completeness, the proof of Theorem 7 is given in Appendix D.

Next, we compare the distributional convergence behavior of the convolution integrals. Since the conditions (8) and (21) are the same, i.e., since $A_{N}^{T} f$ converges pointwise for all $f \in \mathcal{P} \mathcal{W}_{\pi}^{1}$ if and only if $B_{N}^{T} f$ converges uniformly on all of $\mathbb{R}$ for all $f \in$ $\mathcal{P} \mathcal{W}_{\pi}^{1}$, we can combine Theorem 2 and Theorem 5 to obtain the following interesting result about the distributional convergence behavior of the convolution integrals $A_{N}^{T} f$ and $B_{N}^{T} f$.

Corollary 3: Let $T: \mathcal{P} \mathcal{W}_{\pi}^{1} \rightarrow \mathcal{P} \mathcal{W}_{\pi}^{1}$ be a stable LTI system. The following statements are equivalent.

i) $A_{N}^{T} f$ converges in $\mathcal{S}^{\prime}$ for all $f \in \mathcal{P} \mathcal{W}_{\pi}^{1}$.

ii) $A_{N}^{T} f$ converges in $\mathcal{D}^{\prime}$ for all $f \in \mathcal{P} \mathcal{W}_{\pi}^{1}$.

iii) $B_{N}^{T} f$ converges in $\mathcal{S}^{\prime}$ for all $f \in \mathcal{P} \mathcal{W}_{\pi}^{1}$.

iv) $B_{N}^{T} f$ converges in $\mathcal{D}^{\prime}$ for all $f \in \mathcal{P} \mathcal{W}_{\pi}^{1}$.

Corollary 3 shows that both convolution integrals $A_{N}^{T} f$ and $B_{N}^{T} f$ have the same distributional convergence behavior. Thus, there is a difference between the classical convergence behavior of the convolution integrals and the distributional convergence behavior. In the classical setting, the integrals (2) and (3) exhibit a different convergence behavior, whereas in the distributional setting we do not have to distinguish between the integrals, because both have the same convergence behavior.

\section{Convolution Sum}

The discrete counterpart of the convolution integral (2), which is given by the convolution sum (4), naturally emerges from the finite Shannon sampling series

$$
\left(S_{N} f\right)(t):=\sum_{k=-N}^{N} f(k) \operatorname{sinc}(t-k)
$$

when some LTI operator $T$ is applied, because

$$
\begin{aligned}
\left(T S_{N} f\right)(t) & =\sum_{k=-N}^{N} f(k)(T \operatorname{sinc}(\cdot-k))(t) \\
& =\sum_{k=-N}^{N} f(k) h_{T}(t-k)=\left(S_{N}^{T} f\right)(t) .
\end{aligned}
$$

The sum in (4) is important for practical applications because it uses only the samples $\{f(k)\}_{k \in \mathbb{Z}}$ of the signal $f$. If $\left(S_{N}^{T} f\right)(t)$ converges to $(T f)(t)$ for all $t \in \mathbb{R}$ as $N$ tends to infinity, then $\left(S_{N}^{T} f\right)(t)$ can be used to approximate $(T f)(t)$. Of course the convergence of $\left(S_{N}^{T} f\right)(t)$ is not guaranteed and depends on the signal $f$ and the stable LTI system $T$.

For signals in $\mathcal{P} \mathcal{W}_{\pi}^{2}$ the situation is simple, because we have

$$
(T f)(t)=\sum_{k=-\infty}^{\infty} f(k) h_{T}(t-k)
$$

for all stable LTI systems $T: \mathcal{P W}_{\pi}^{2} \rightarrow \mathcal{P} \mathcal{W}_{\pi}^{2}$ and all signals $f \in \mathcal{P} \mathcal{W}_{\pi}^{2}$. This is due to the convergence of the Shannon sam-

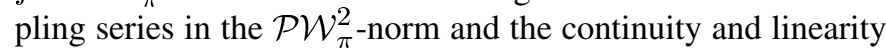
of $T$.

Unfortunately, for signals $f \in \mathcal{P} \mathcal{W}_{\pi}^{1}$ and stable LTI systems $T$ operating on $\mathcal{P W}_{\pi}^{1},\left(S_{N}^{T} f\right)(t)$ does not always converge to $(T f)(t)$. There are stable LTI systems $T$ for which $\left(S_{N}^{T} f\right)(t)$ diverges for some signal $f \in \mathcal{P W}_{\pi}^{1}$ [20]. In part i) of Theorem 8 we characterize the stable LTI systems $T$ for which $\left(S_{N}^{T} f\right)(t)$ converges pointwise to $(T f)(t)$ for all $f \in \mathcal{P} \mathcal{W}_{\pi}^{1}$.

Further, we analyze the distributional convergence behavior of $S_{N}^{T} f$. For this purpose we introduce the abbreviation

$$
S_{N, \phi}^{T} f:=\int_{-\infty}^{\infty}\left(S_{N}^{T} f\right)(t) \phi(t) \mathrm{d} t .
$$

In part ii) of Theorem 8 we characterize the stable LTI systems $T$ for which the convolution sum $S_{N}^{T} f$ converges in $\mathcal{S}^{\prime}$ for all $f \in \mathcal{P} \mathcal{W}_{\pi}^{1}$.

Theorem 8: Let $T: \mathcal{P} \mathcal{W}_{\pi}^{1} \rightarrow \mathcal{P} \mathcal{W}_{\pi}^{1}$ be a stable LTI system.

i) For all $t \in \mathbb{R}$ and all $f \in \mathcal{P} \mathcal{W}_{\pi}^{1}$ we have

$$
\lim _{N \rightarrow \infty}\left|(T f)(t)-\left(S_{N}^{T} f\right)(t)\right|=0
$$

if and only if there exists a constant $C_{8}=C_{8}(t)<\infty$ such that

$$
\max _{\omega \in[-\pi, \pi]}\left|\frac{1}{2 \pi} \int_{-\pi}^{\pi} \hat{h}_{T}\left(\omega_{1}\right) \mathrm{e}^{i \omega_{1} t} \frac{\sin \left[\left(N+\frac{1}{2}\right)\left(\omega-\omega_{1}\right)\right]}{\sin \left(\frac{\omega-\omega_{1}}{2}\right)} \mathrm{d} \omega_{1}\right|
$$$$
\leq C_{8}(t)
$$

for all $N \in \mathbb{N}$. In addition, if (25) is not fulfilled, then there exists a signal $f_{1} \in \mathcal{P} \mathcal{W}_{\pi}^{1}$ such that

$$
\limsup _{N \rightarrow \infty}\left|\left(S_{N}^{T} f_{1}\right)(t)\right|=\infty \text {. }
$$


ii) Moreover, we have

$$
\lim _{N \rightarrow \infty}\left|S_{N, \phi}^{T} f-\int_{-\infty}^{\infty}(T f)(t) \phi(t) \mathrm{d} t\right|=0
$$

for all $f \in \mathcal{P} \mathcal{W}_{\pi}^{1}$ and all $\phi \in \mathcal{S}$ if and only if for all $\phi \in \mathcal{S}$ there exists a constant $C_{9}=C_{9}(\phi)<\infty$ such that

$$
\max _{\omega \in[-\pi, \pi]}\left|\frac{1}{2 \pi} \int_{-\pi}^{\pi} \hat{h}_{T}\left(\omega_{1}\right) \hat{\phi}\left(-\omega_{1}\right) \frac{\sin \left[\left(N+\frac{1}{2}\right)\left(\omega-\omega_{1}\right)\right]}{\sin \left(\frac{\omega-\omega_{1}}{2}\right)} \mathrm{d} \omega_{1}\right|
$$

for all $N \in \mathbb{N}$. In addition, if (26) is not fulfilled for some $\phi \in \mathcal{S}$, then there exists a signal $f_{1} \in \mathcal{P} \mathcal{W}_{\pi}^{1}$ such that

$$
\limsup _{N \rightarrow \infty}\left|S_{N, \phi}^{T} f_{1}\right|=\infty \text {. }
$$

Part i) of Theorem 8 was proven in [20], and the proof of part ii) is done analogously to the proof of part ii) of Theorem 1 .

Like the proofs of the Theorems 1 and 4, the proof of Theorem 8 does not rely on the fact that $\phi \in \mathcal{S}$. All arguments also hold if $\phi \in \mathcal{D}$. This observation leads to the following corollary about the convergence of $S_{N}^{T} f$ in $\mathcal{D}^{\prime}$.

Corollary 4: Part ii) of Theorem 8 remains true if $\phi \in \mathcal{S}$ is replaced with $\phi \in \mathcal{D}$.

In Theorem 11 we will use the characterization that is provided by Corollary 4 to show that there exists a stable LTI system $T_{1}$ for which $S_{N}^{T_{1}} f_{1}$ diverges in $\mathcal{D}^{\prime}$ for some $f_{1} \in \mathcal{P} \mathcal{W}_{\pi}^{1}$.

\section{DifFERENCE BetweEn the Convolution INTEGRAL AND THE CONVOLUTION SUM}

In Theorem 1 we have the special situation that the convergence of $\left(A_{N}^{T} f\right)(t)$ for one $t \in \mathbb{R}$ and all $f \in \mathcal{P} \mathcal{W}_{\pi}^{1}$ implies the convergence of $\left(A_{N}^{T} f\right)(t)$ for all $t \in \mathbb{R}$ and all $f \in \mathcal{P} \mathcal{W}_{\pi}^{1}$. In this section we investigate the question whether the convolution sum exhibits the same behavior, i.e., whether the convergence of $\left(S_{N}^{T} f\right)(t)$ for one $t \in \mathbb{R}$ and all $f \in \mathcal{P W}_{\pi}^{1}$ implies the convergence of $\left(S_{N}^{T} f\right)(t)$ for all $t \in \mathbb{R}$ and all $f \in \mathcal{P} \mathcal{W}_{\pi}^{1}$. Despite the obvious similarities between the convolution integral and the convolution sum, the surprising to this question is no.

Theorem 9: For every $t \in \mathbb{R}$ and every $t^{*} \in \mathbb{R} \backslash(t+\mathbb{Z})$ there exists a stable LTI system $T_{1}: \mathcal{P} \mathcal{W}_{\pi}^{1} \rightarrow \mathcal{P} \mathcal{W}_{\pi}^{1}$ such that

$$
\lim _{N \rightarrow \infty}\left|\left(T_{1} f\right)(t)-\left(S_{N}^{T_{1}} f\right)(t)\right|=0
$$

for all $f \in \mathcal{P} \mathcal{W}_{\pi}^{1}$ and

$$
\limsup _{N \rightarrow \infty}\left(S_{N}^{T_{1}} f_{1}\right)\left(t^{*}\right)=\infty
$$

for some $f_{1} \in \mathcal{P} \mathcal{W}_{\pi}^{1}$.
Proof: Let $t \in \mathbb{R}$ and $t^{*} \in \mathbb{R} \backslash(t+\mathbb{Z})$ be arbitrary but fixed. According to Theorem 8 i) and the equality

$$
\begin{array}{r}
\frac{1}{2 \pi} \int_{-\pi}^{\pi} \hat{h}_{T}\left(\omega_{1}\right) \mathrm{e}^{i \omega_{1} t} \frac{\sin \left[\left(N+\frac{1}{2}\right)\left(\omega-\omega_{1}\right)\right]}{\sin \left(\frac{\omega-\omega_{1}}{2}\right)} \mathrm{d} \omega_{1} \\
=\sum_{k=-N}^{N} h_{T}(t-k) \mathrm{e}^{i \omega k},
\end{array}
$$

we have (27) for all $f \in \mathcal{P} \mathcal{W}_{\pi}^{1}$ if and only if

$$
\sup _{N \in \mathbb{N} \omega \in[-\pi, \pi]} \max _{k=-N}\left|\sum_{k}^{N} h_{T_{1}}(t-k) \mathrm{e}^{i \omega k}\right|<\infty .
$$

Furthermore, we have (28) for some $f_{1} \in \mathcal{P} \mathcal{W}_{\pi}^{1}$ if and only if

$$
\limsup _{N \rightarrow \infty} \max _{\omega \in[-\pi, \pi]}\left|\sum_{k=-N}^{N} h_{T_{1}}\left(t^{*}-k\right) \mathrm{e}^{i \omega k}\right|=\infty .
$$

Thus, we have to show that there exists a stable LTI system $T_{1}$ such that (29) and (30) is true.

To this end, we consider the space $\mathcal{K}$ that consists of all functions $h$ with a representation $h(\tau)=1 /(2 \pi) \int_{-\pi}^{\pi} \hat{h}(w) \mathrm{e}^{i \omega \tau} \mathrm{d} \omega$, $t \in \mathbb{R}$, for some $\hat{h} \in C[-\pi, \pi]$ and with finite norm

$$
\|h\|_{\mathcal{K}, t}:=\|\hat{h}\|_{\infty}+\sup _{N \in \mathbb{N}} \max _{\omega \in[-\pi, \pi]}\left|\sum_{k=-N}^{N} h(t-k) \mathrm{e}^{i \omega k}\right| .
$$

The space $\mathcal{K}$, equipped with the norm $\|\cdot\|_{\mathcal{K}, t}$ is a Banach space. Next, we consider the sequence of bounded linear operators $\left\{U_{N}\right\}_{N \in \mathbb{N}}$ that map $\mathcal{K}$ into $\left(C[-\pi, \pi],\|\cdot\|_{L^{\infty}[-\pi, \pi]}\right)$, defined by

$$
\left(U_{N} h\right)(\omega)=\sum_{k=-N}^{N} h\left(t^{*}-k\right) \mathrm{e}^{i \omega k} .
$$

Further, we need the functions $h_{n}, n \in \mathbb{N}$, given by

$$
h_{n}(\tau)=\frac{\sin (\pi(\tau-n-t))}{2 \pi(\tau-n-t)} .
$$

Since

$$
\left\|h_{n}\right\|_{\mathcal{K}, t} \frac{1}{2}+\sup _{N \in \mathbb{N}} \max _{\omega \in[-\pi, \pi]}\left|\sum_{k=-N}^{N} \frac{\sin (\pi(k+n))}{2 \pi(k+n)} \mathrm{e}^{i \omega k}\right|=1,
$$

we have

$$
\begin{aligned}
\left\|U_{N}\right\| & =\sup _{\|h\|_{\mathcal{K}, t \leq 1}} \max _{\omega \in[-\pi, \pi]}\left|\left(U_{N} h\right)(\omega)\right| \\
& \geq \max _{\omega \in[-\pi, \pi]}\left|\left(U_{N} h_{N}\right)(\omega)\right| \\
& =\max _{\omega \in[-\pi, \pi]}\left|\sum_{k=-N}^{N} \frac{(-1)^{k+N} \sin \left(\pi\left(t^{*}-t\right)\right)}{2 \pi\left(t^{*}-t-k-N\right)} \mathrm{e}^{i \omega k}\right| \\
& \geq\left|\frac{\sin \left(\pi\left(t^{*}-t\right)\right)}{2 \pi} \sum_{k=-N}^{N} \frac{1}{t^{*}-t-k-N}\right|
\end{aligned}
$$


for all $N \in \mathbb{N}$. Moreover, we have that

$$
\lim _{N \rightarrow \infty}\left|\sum_{k=-N}^{N} \frac{1}{t^{*}-t-k-N}\right|=\infty
$$

which implies that $\sup _{N \in \mathbb{N}}\left\|U_{N}\right\|=\infty$. Thus, according to the Banach-Steinhaus theorem [18, p. 98] there exists a function $h_{T_{1}} \in \mathcal{K}$ such that

$$
\begin{aligned}
& \limsup _{N \rightarrow \infty}\left\|U_{N} h_{T_{1}}\right\|_{L^{\infty}[-\pi, \pi]} \\
&=\limsup _{N \rightarrow \infty} \max _{\omega \in[-\pi, \pi]}\left|\sum_{k=-N}^{N} h_{T_{1}}\left(t^{*}-k\right) \mathrm{e}^{i \omega k}\right|=\infty .
\end{aligned}
$$

By the definition of the space $\mathcal{K}, h_{T_{1}}$ fulfills

$$
\sup _{N \in \mathbb{N}} \max _{\omega \in[-\pi, \pi]}\left|\sum_{k=-N}^{N} h_{T_{1}}(t-k) \mathrm{e}^{i \omega k}\right|<\infty .
$$

So $T_{1}$ is the desired stable LTI system.

In Section VII-B, we will illustrate Theorem 9 with a numerical example that shows the behavior of $\left\|U_{N}\right\|$.

According to Theorem 9 we cannot conclude the convergence of $\left(S_{N}^{T} f\right)(t)$ for all $t \in \mathbb{R}$ and all $f \in \mathcal{P} \mathcal{W}_{\pi}^{1}$ from the convergence of $\left(S_{N}^{T} f\right)(t)$ for some fixed $t \in \mathbb{R}$ and all $f \in \mathcal{P} \mathcal{W}_{\pi}^{1}$. This is in contrast to the situation in Section IV-A, where exactly this was possible. Consequently, for $\left(S_{N}^{T} f\right)(t)$ we cannot obtain an equivalence like the equivalence between item iii) and item iv) in Theorem 2.

Nevertheless, it would be satisfying if the following convergence types could be set in relation:

S1) $S_{N}^{T} f$ converges in $\mathcal{D}^{\prime}$ for all $f \in \mathcal{P} \mathcal{W}_{\pi}^{1}$;

S2) $S_{N}^{T} f$ converges in $\mathcal{S}^{\prime}$ for all $f \in \mathcal{P W}_{\pi}^{1}$;

S3) $\left(S_{N}^{T} f\right)(t)$ converges pointwise for all $t \in \mathbb{R}$ and all $f \in$ $\mathcal{P} \mathcal{W}_{\pi}^{1}$

S4) $\left(S_{N}^{T} f\right)(t)$ converges uniformly on all compact subsets of $\mathbb{R}$ for all $f \in \mathcal{P} \mathcal{W}_{\pi}^{1}$.

In general, the analysis of the convolution $\operatorname{sum}\left(S_{N}^{T} f\right)(t)$ is more intricate than the analysis of the convolution integral, because of the periodicity of the Dirichlet kernel

$$
\frac{\sin \left[\left(N+\frac{1}{2}\right)(\omega)\right]}{\sin \left(\frac{\omega}{2}\right)} .
$$

We do not fully know the relation between S1, S2, S3, and S4. However, we have the following connections.

Theorem 10: Let $T: \mathcal{P} \mathcal{W}_{\pi}^{1} \rightarrow \mathcal{P} \mathcal{W}_{\pi}^{1}$ be a stable LTI system.

i) $\mathrm{S} 2$ implies $\mathrm{S} 1$.

ii) $\mathrm{S} 2$ implies $\mathrm{S} 3$.

iii) S4 implies S1.

Proof:

i) Observation 1.

ii) Let $t \in \mathbb{R}$ be arbitrary but fixed. Since $S_{N}^{T} f$ converges in $\mathcal{S}^{\prime}$ for all $f \in \mathcal{P} \mathcal{W}_{\pi}^{1}$ there exists, according to part ii) of Theorem 8, for every $\phi \in \mathcal{S}$ a constant $C_{9}(\phi)$ such that
(26) is true for all $N \in \mathbb{N}$. For the specific $\phi_{1} \in \mathcal{S}$ with $\hat{\phi}_{1}(\omega)=\mathrm{e}^{-i \omega t}$ for $\omega \in[-\pi, \pi]$ we obtain

$$
\begin{aligned}
& \max _{\omega \in[-\pi, \pi]}\left|\frac{1}{2 \pi} \int_{-\pi}^{\pi} \hat{h}_{T}\left(\omega_{1}\right) \mathrm{e}^{i \omega_{1} t} \frac{\sin \left[\left(N+\frac{1}{2}\right)\left(\omega-\omega_{1}\right)\right]}{\sin \left(\frac{\omega-\omega_{1}}{2}\right)} \mathrm{d} \omega_{1}\right| \\
& \leq C_{9}\left(\phi_{1}\right)
\end{aligned}
$$

for all $N \in \mathbb{N}$. Thus, the assertion follows from part i) of Theorem 8 .

iii) Let $\phi \in \mathcal{D}$ be arbitrary but fixed. Since $\phi$ is concentrated on some compact set $I \subset \mathbb{R}$, we have

$$
S_{N, \phi}^{T} f=\int_{-\infty}^{\infty}\left(S_{N}^{T} f\right)(t) \phi(t) \mathrm{d} t=\int_{I}\left(S_{N}^{T} f\right)(t) \phi(t) \mathrm{d} t .
$$

It follows that

$$
\begin{aligned}
\lim _{N \rightarrow \infty} S_{N, \phi}^{T} f & =\int_{I} \lim _{N \rightarrow \infty}\left(S_{N}^{T} f\right)(t) \phi(t) \mathrm{d} t=\int_{I} f(t) \phi(t) \mathrm{d} t \\
& =\int_{-\infty}^{\infty} f(t) \phi(t) \mathrm{d} t
\end{aligned}
$$

because $\left(S_{N}^{T} f\right)(t)$ converges uniformly on $I$ by assumption. Since $\phi \in \mathcal{D}$ was arbitrary, the proof is complete.

From the results in [20] it can be seen that there exists a stable LTI system $T_{1}$ such that $\left(S_{N}^{T_{1}} f_{1}\right)(t)$ diverges for some $t \in \mathbb{R}$ and $f_{1} \in \mathcal{P} \mathcal{W}_{\pi}^{1}$. Hence, item ii) of Theorem 10 implies that $S_{N}^{T_{1}} f_{1}$ diverges in $\mathcal{S}^{\prime}$ for some $f_{1} \in \mathcal{P} \mathcal{W}_{\pi}^{1}$. This shows that there are stable LTI systems for which the convolution sum diverges even in $\mathcal{S}^{\prime}$. However, since we do not know whether S1 implies S3, we cannot immediately conclude the divergence of $S_{N}^{T_{1}} f_{1}$ in $\mathcal{D}^{\prime}$. Anyhow, the following theorem shows that we have this divergence.

Theorem 11: There exists a stable LTI system $T_{1}: \mathcal{P} \mathcal{W}_{\pi}^{1} \rightarrow$ $\mathcal{P} \mathcal{W}_{\pi}^{1}$ and a signal $f_{1} \in \mathcal{P} \mathcal{W}_{\pi}^{1}$ such that

$$
\limsup _{N \rightarrow \infty}\left|S_{N, \phi_{1}}^{T_{1}} f_{1}\right|=\infty
$$

for some $\phi_{1} \in \mathcal{D}$.

Proof: Using the characterization that was provided by Corollary 4, we have to show that there exists a stable LTI system $T_{1}$ and a function $\phi_{1} \in \mathcal{D}$ such that

$$
\begin{aligned}
\sup _{N \in \mathbb{N}} \max _{\omega \in[-\pi, \pi]} \mid \frac{1}{2 \pi} \int_{-\pi}^{\pi} \hat{h}_{T_{1}}\left(\omega_{1}\right) \hat{\phi}_{1}\left(-\omega_{1}\right) \times & \\
& \frac{\sin \left[\left(N+\frac{1}{2}\right)\left(\omega-\omega_{1}\right)\right]}{\sin \left(\frac{\omega-\omega_{1}}{2}\right)} \mathrm{d} \omega_{1} \mid=\infty .
\end{aligned}
$$

Let

$$
\hat{h}_{T, N}\left(\omega_{1}\right)=\operatorname{sgn}\left(\frac{\sin \left[\left(N+\frac{1}{2}\right)\left(\omega_{1}\right)\right]}{\sin \left(\frac{\omega_{1}}{2}\right)}\right)
$$


and choose some $\phi_{1} \in \mathcal{D}$ such that $\hat{\phi}_{1}$ is real valued and $\hat{\phi}_{1}(\omega) \geq 1$ for all $\omega \in[-\pi, \pi]$. Next, we analyze the sequence of bounded linear functionals $K_{N}: L^{\infty}[-\pi, \pi] \rightarrow \mathbb{C}, N \in \mathbb{N}$, given by

$$
K_{N} f=\frac{1}{2 \pi} \int_{-\pi}^{\pi} f\left(\omega_{1}\right) \hat{\phi}_{1}\left(-\omega_{1}\right) \frac{\sin \left[\left(N+\frac{1}{2}\right)\left(\omega_{1}\right)\right]}{\sin \left(\frac{\omega_{1}}{2}\right)} \mathrm{d} \omega_{1} .
$$

For $N \in \mathbb{N}$, we have

$$
\begin{aligned}
\left|K_{N} \hat{h}_{T, N}\right| & =\frac{1}{2 \pi} \int_{-\pi}^{\pi} \hat{\phi}_{1}\left(-\omega_{1}\right)\left|\frac{\sin \left[\left(N+\frac{1}{2}\right)\left(\omega_{1}\right)\right]}{\sin \left(\frac{\omega_{1}}{2}\right)}\right| \mathrm{d} \omega_{1} \\
& \geq \frac{1}{2 \pi} \int_{-\pi}^{\pi}\left|\frac{\sin \left[\left(N+\frac{1}{2}\right)\left(\omega_{1}\right)\right]}{\sin \left(\frac{\omega_{1}}{2}\right)}\right| \mathrm{d} \omega_{1} \\
& \geq \frac{2}{\pi^{2}} \log (1+N)
\end{aligned}
$$

where the last inequality follows from the well-known divergence of the $L^{1}$-norm of the Dirichlet kernel [18, p. 102]. It follows that

$\left\|K_{N}\right\|=\sup _{\left\|\hat{h}_{T}\right\|_{L} \infty[-\pi, \pi]} \leq 1 \leq K_{N} \hat{h}_{T}|\geq| K_{N} \hat{h}_{T, N} \mid \geq \frac{2}{\pi^{2}} \log (1+N)$

for all $N \in \mathbb{N}$, and consequently that $\sup _{N \in \mathbb{N}}\left\|K_{N}\right\|=\infty$. Thus, the Banach-Steinhaus theorem [18, p. 98] implies that there exists a $\hat{h}_{T_{1}} \in L^{\infty}[-\pi, \pi]$ such that

$\sup _{N \in \mathbb{N}}\left|\frac{1}{2 \pi} \int_{-\pi}^{\pi} \hat{h}_{T_{1}}\left(\omega_{1}\right) \hat{\phi}_{1}\left(-\omega_{1}\right) \frac{\sin \left[\left(N+\frac{1}{2}\right)\left(\omega_{1}\right)\right]}{\sin \left(\frac{\omega_{1}}{2}\right)} \mathrm{d} \omega_{1}\right|=\infty$.

The proof is complete, because (33) implies (32).

Although we cannot say whether S3 is a necessary condition for S1, we see from Theorem 11 that there are stable LTI systems and signals in $\mathcal{P} \mathcal{W}_{\pi}^{1}$ such that the convolution sum (4) diverges in $\mathcal{D}^{\prime}$. In this regard, we have the same situation as in Section IV, where we analyzed the convolution integral: The divergence of the convolution sum in the classical, nondistributional setting cannot be circumvented by considering the more relaxed concept of distributional convergence. Therefore, a convolution type representation of stable LTI systems in the form (4) is not possible in general for the space $\mathcal{P} \mathcal{W}_{\pi}^{1}$, even if the convergence is treated in the distributional sense.

\section{NUMERICAL EXAMPLE}

\section{A. Example 1: Convolution Integral}

In this section we numerically illustrate the divergence of the convolution integral (2). We use the same system that was con-

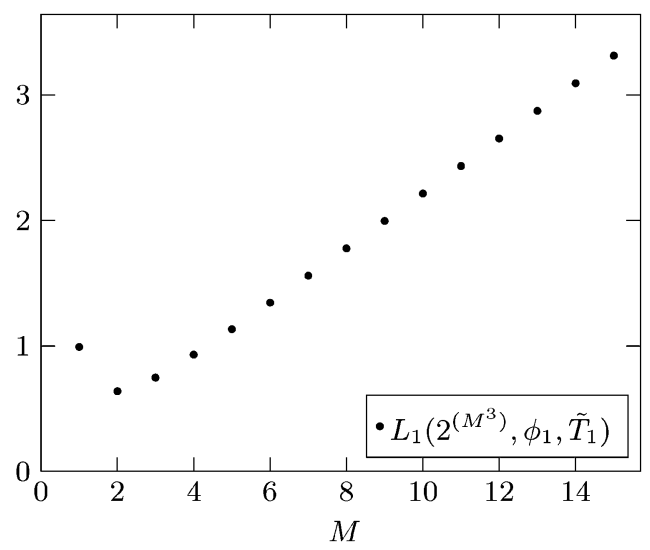

Fig. 1. Divergence of $L_{1}\left(2^{\left(M^{3}\right)}, \phi_{1}, \tilde{T}_{1}\right)$.

structed in the proof of Theorem 3. In Theorem 1, we have seen that

$$
\begin{aligned}
& L_{1}(N, \phi, T) \\
& \quad:=\left|\frac{1}{\pi} \int_{-\pi}^{\pi} \hat{h}_{T}\left(\omega_{1}\right) \hat{\phi}\left(-\omega_{1}\right) \frac{\sin \left(N\left(\omega_{1}\right)\right)}{\omega_{1}} \mathrm{~d} \omega_{1}\right| \\
& \quad \leq \max _{\omega \in[-\pi, \pi]}\left|\frac{1}{\pi} \int_{-\pi}^{\pi} \hat{h}_{T}\left(\omega_{1}\right) \hat{\phi}\left(-\omega_{1}\right) \frac{\sin \left(N\left(\omega-\omega_{1}\right)\right)}{\omega-\omega_{1}} \mathrm{~d} \omega_{1}\right|
\end{aligned}
$$

plays an important role for the convergence of $A_{N}^{T} f$. $L_{1}(N, \phi, T)$ has to be uniformly bounded with respect to $N$ for all $\phi \in \mathcal{S}$ in order that $A_{N}^{T} f$ converges in $\mathcal{S}^{\prime}$ for all $f \in \mathcal{P W}_{\pi}^{1}$. Otherwise, there exists a signal $f_{1} \in \mathcal{P} \mathcal{W}_{\pi}^{1}$ such that $A_{N}^{T} f_{1}$ diverges in $\mathcal{S}^{\prime}$.

Next, we illustrate the divergence of $L_{1}\left(N, \phi_{1}, T_{1}\right)$ for the stable LTI system $T_{1}$, given by $\hat{h}_{T_{1}}(\omega)=$ $\sum_{k=1}^{\infty} k^{-2} \sin \left(2^{\left(k^{3}\right)} \omega\right) \operatorname{sgn}(w)$ for $\omega \in[-\pi, \pi]$, and the function $\phi_{1} \in \mathcal{S}$ with $\hat{\phi}_{1}(\omega)=1$ for $\omega \in[-\pi, \pi]$. To this end, we consider the approximation $\hat{h}_{\tilde{T}_{1}}(\omega)=\sum_{k=1}^{15} k^{-2} \sin \left(2^{\left(k^{3}\right)} \omega\right) \operatorname{sgn}(w)$ of $\hat{h}_{T_{1}}$. We cannot use $\hat{h}_{T_{1}}$ directly, because of computational complexity reasons. In the simulation we evaluate $L_{1}\left(2^{\left(M^{3}\right)}, \phi_{1}, \tilde{T}_{1}\right)$ for $M=1, \ldots, 15$. The increase of $L_{1}\left(2^{\left(M^{3}\right)}, \phi_{1}, \tilde{T}_{1}\right)$ is clearly visible in Fig. 1.

\section{B. Example 2: Convolution Sum}

Our second numerical example illustrates the behavior of the convolution sum (4). The important element in the proof of Theorem 9 was the norm $\left\|U_{N}\right\|$. Since

||$U_{N} \| \geq\left|\left(U_{N} h_{N}\right)(\pi)\right|=\left|\sum_{k=-N}^{N} h_{N}\left(t^{*}-k\right) \mathrm{e}^{i \pi k}\right|:=L_{2}\left(N, t^{*}\right)$,

we numerically evaluate $L_{2}\left(N, t^{*}\right)$ for

$$
h_{N}(\tau)=\frac{\sin (\pi(\tau-N-t))}{2 \pi(\tau-N-t)} .
$$

Without loss of generality we set $t=0$ in the simulation. According to the theory, we expect $L_{2}\left(N, t^{*}\right)$ to be uniformly 


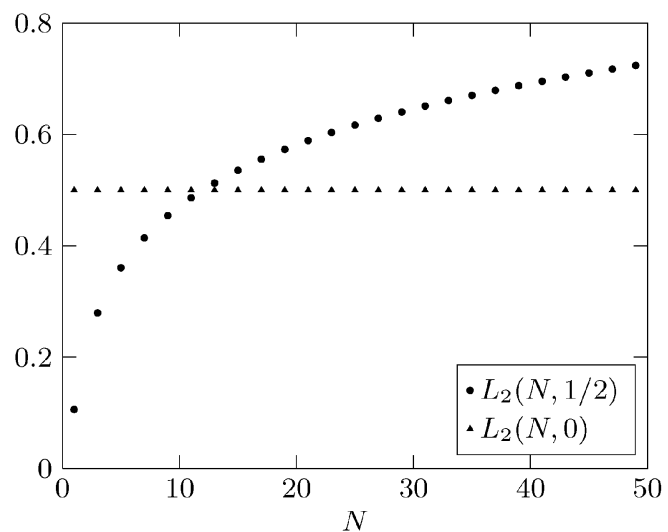

Fig. 2. Plot of $L_{2}(N, 1 / 2)$ and $L_{2}(N, 0)$.

bounded with respect to $N$ for all $t^{*} \in \mathbb{Z}$, and divergent for all $t^{*} \in \mathbb{R} \backslash \mathbb{Z}$. In Fig. 2 it can be seen that $L_{2}(N, 0)$ is constant $1 / 2$, independently of $N$, and that $L_{2}(N, 1 / 2)$ increases with increasing $N$.

\section{CONCLUSION}

In this work, we analyzed the convergence behavior of two commonly used time-domain convolution type system representations for the Paley-Wiener space $\mathcal{P} \mathcal{W}_{\pi}^{1}$. Although the convolution integrals have a different classical convergence behavior, it turned out that they have the same distributional convergence behavior. Unfortunately, there exist stable LTI systems and signals for which the convolution integrals diverge even in a distributional sense. Hence, the more relaxed concept of distributional convergence cannot circumvent the convergence problems of the convolution integrals, encountered in the classical, nondistributional setting. This results is interesting because it shows that a convolution type time-domain representation of stable LTI systems operating on $\mathcal{P} \mathcal{W}_{\pi}^{1}$ is not always possible, even though such systems always have a frequency-domain representation. Further, we completely characterized all stable LTI systems for which a convolution type system representation is possible.

Although the convergence of the analyzed convolution type system representations (2)-(4) is problematic, it is not obvious what other-more complicated-representations exist, which are convergent for all stable LTI systems and all signals in $\mathcal{P W}_{\pi}^{1}$. To find such representations, especially for important systems like the Hilbert transform, would be a challenging task for further research.

\section{APPENDIX}

\section{A. An Auxiliary Lemma}

The following lemma is needed for several proofs in the appendix.

Lemma 4: For the operator $U: \mathcal{P W}_{\pi}^{1} \rightarrow \mathbb{C}$ defined by $U f=$ $1 /(2 \pi) \int_{-\pi}^{\pi} \hat{f}(\omega) K(\omega) \mathrm{d} \omega$, where $K \in L^{\infty}[-\pi, \pi]$, we have $\sup _{\|f\|_{\mathcal{P} \mathcal{W}^{1}} \leq 1}|U f|=\|K\|_{L^{\infty}[-\pi, \pi]}$.

Proof: Lemma 4 is a direct consequence of Lemma 17 in [21].

\section{B. Proof of Lemma 1}

Proof: Let $\phi \in \mathcal{S}, N \in \mathbb{N}$, and the stable LTI systems $T$ be arbitrary but fixed. For $f \in \mathcal{P} \mathcal{W}_{\pi}^{1}$ we have

$$
\begin{aligned}
A_{N, \phi}^{T} f & =\int_{-\infty}^{\infty} \int_{-N}^{N} f(\tau) h_{T}(t-\tau) \phi(t) \mathrm{d} \tau \mathrm{d} t \\
& =\int_{-\infty}^{\infty} \int_{-N}^{N} \frac{1}{2 \pi} \int_{-\pi}^{\pi} \hat{f}(\omega) \mathrm{e}^{i \omega \tau} h_{T}(t-\tau) \phi(t) \mathrm{d} \omega \mathrm{d} \tau \mathrm{d} t \\
& =\frac{1}{2 \pi} \int_{-\pi}^{\pi} \hat{f}(\omega) \int_{-N}^{N} \mathrm{e}^{i \omega \tau} \int_{-\infty}^{\infty} h_{T}(t-\tau) \phi(t) \mathrm{d} t \mathrm{~d} \tau \mathrm{d} \omega
\end{aligned}
$$

where the order of the integrals was interchanged according to Fubini's theorem, which is applicable because

$$
\begin{aligned}
& \int_{-\infty}^{\infty} \int_{-N}^{N} \frac{1}{2 \pi} \int_{-\pi}^{\pi}\left|\hat{f}(\omega) \mathrm{e}^{i \omega \tau} h_{T}(t-\tau) \phi(t)\right| \mathrm{d} \omega \mathrm{d} \tau \mathrm{d} t \\
& \leq 2 N\left\|h_{T}\right\|_{\infty} \int_{-\infty}^{\infty} \frac{1}{2 \pi} \int_{-\pi}^{\pi}|\hat{f}(\omega) \phi(t)| \mathrm{d} \omega \mathrm{d} t \\
& \leq 2 N\|T\|\|f\|_{\mathcal{P} \mathcal{W}_{\pi}^{1}}\|\phi\|_{1}<\infty .
\end{aligned}
$$

Moreover, since $h_{T} \in L^{2}$ and $\phi \in L^{2}$, we obtain

$$
\int_{-\infty}^{\infty} h_{T}(t-\tau) \phi(t) \mathrm{d} t=\frac{1}{2 \pi} \int_{-\pi}^{\pi} \hat{h}_{T}\left(\omega_{1}\right) \hat{\phi}\left(-\omega_{1}\right) \mathrm{e}^{-i \omega_{1} \tau} \mathrm{d} \omega_{1}
$$

by applying the generalized Parseval equality. Thus, it follows that

$$
\begin{aligned}
A_{N, \phi}^{T} f=\frac{1}{2 \pi} \int_{-\pi}^{\pi} \hat{f}(\omega) \frac{1}{\pi} \int_{-\pi}^{\pi} \hat{h}_{T}\left(\omega_{1}\right) \hat{\phi}\left(-\omega_{1}\right) \\
\times \frac{\sin \left(N\left(\omega-\omega_{1}\right)\right)}{\omega-\omega_{1}} \mathrm{~d} \omega_{1} \mathrm{~d} \omega .
\end{aligned}
$$

\section{Proof of Lemma 2}

Proof: First, we derive a preliminary statement, which will be used in the proof of i) and ii). For $\omega \in[-\pi, \pi]$ and $\phi \in \mathcal{S}$ consider the difference

$$
\begin{aligned}
& D_{N}\left(\hat{h}_{T}, \phi, \omega\right) \\
&:=\mid \frac{1}{\pi} \int_{-\pi}^{\pi} \hat{h}_{T}\left(\omega_{1}\right) \hat{\phi}\left(-\omega_{1}\right) \frac{\sin \left(N\left(\omega-\omega_{1}\right)\right)}{\omega-\omega_{1}} \mathrm{~d} \omega_{1} \\
& \quad \quad-\hat{\phi}(-\omega) \frac{1}{\pi} \int_{-\pi}^{\pi} \hat{h}_{T}\left(\omega_{1}\right) \frac{\sin \left(N\left(\omega-\omega_{1}\right)\right)}{\omega-\omega_{1}} \mathrm{~d} \omega_{1} \mid \\
&=\mid \frac{1}{\pi} \int_{-\pi}^{\pi} \hat{h}_{T}\left(\omega_{1}\right) \frac{\hat{\phi}\left(-\omega_{1}\right)-\hat{\phi}(-\omega)}{\omega-\omega_{1}} \\
& \quad \times \sin \left(N\left(\omega-\omega_{1}\right)\right) \mathrm{d} \omega_{1} \mid .
\end{aligned}
$$


Since $\phi \in \mathcal{S}$ it follows that $\hat{\phi} \in \mathcal{S}$ and $\hat{\phi}^{\prime} \in \mathcal{S}$. In particular, $\phi$ is Lipschitz continuous because $\left\|\hat{\phi}^{\prime}\right\|_{\infty}<\infty$, and we have

$$
\left|\frac{\hat{\phi}\left(-\omega_{1}\right)-\hat{\phi}(-\omega)}{\omega-\omega_{1}}\right| \leq\left\|\hat{\phi}^{\prime}\right\|_{\infty}
$$

for all $\omega \in[-\pi, \pi]$ and $\omega_{1} \in[-\pi, \pi]$. Therefore, we obtain

$$
D_{N}\left(\hat{h}_{T}, \phi, \omega\right) \leq 2\left\|\hat{\phi}^{\prime}\right\|_{\infty}\left\|\hat{h}_{T}\right\|_{L^{\infty}[-\pi, \pi]}=C_{10}\left(\hat{h}_{T}, \phi\right)
$$

for all $\omega \in[-\pi, \pi]$ and all $\phi \in \mathcal{S}$, where $C_{10}\left(\hat{h}_{T}, \phi\right)$ is some positive constant that does not depend $N$. Since $\mathcal{D} \subset \mathcal{S}$, (36) is also true for all $\phi \in \mathcal{D}$.

"i)": Let $N \in \mathbb{N}$ be arbitrary but fixed. Since (11) is true for all $\phi \in \mathcal{D}$ it is in particular true for the specific function $\phi_{1} \in \mathcal{D}$ with real valued $\hat{\phi}_{1}$ and $\hat{\phi}_{1}(\omega)>0$ for $\omega \in[-\pi, \pi]$. Therefore, it follows from (35) and (36) that

$$
\begin{array}{r}
\hat{\phi}_{1}(-\omega)\left|\frac{1}{\pi} \int_{-\pi}^{\pi} \hat{h}_{T}\left(\omega_{1}\right) \frac{\sin \left(N\left(\omega-\omega_{1}\right)\right)}{\omega-\omega_{1}} \mathrm{~d} \omega_{1}\right| \leq C_{10}\left(\hat{h}_{T}, \phi_{1}\right) \\
+\left|\frac{1}{\pi} \int_{-\pi}^{\pi} \hat{h}_{T}\left(\omega_{1}\right) \hat{\phi}_{1}\left(-\omega_{1}\right) \frac{\sin \left(N\left(\omega-\omega_{1}\right)\right)}{\omega-\omega_{1}} \mathrm{~d} \omega_{1}\right|
\end{array}
$$

for all $\omega \in[-\pi, \pi]$. Dividing by $\hat{\phi}_{1}(-\omega)$ and taking the maximum on both sides yields

$$
\begin{aligned}
\max _{\omega \in[-\pi, \pi]}\left|\frac{1}{\pi} \int_{-\pi}^{\pi} \hat{h}_{T}\left(\omega_{1}\right) \frac{\sin \left(N\left(\omega-\omega_{1}\right)\right)}{\omega-\omega_{1}} \mathrm{~d} \omega_{1}\right| \\
\leq M\left(\phi_{1}\right) C_{10}\left(\hat{h}_{T}, \phi_{1}\right)+M\left(\phi_{1}\right) C_{2}\left(\phi_{1}\right)<\infty
\end{aligned}
$$

where $M\left(\phi_{1}\right)=\max _{\omega \in[-\pi, \pi]} 1 / \hat{\phi}_{1}(-\omega)$.

"ii)": Let $N \in \mathbb{N}$ be arbitrary but fixed. Suppose (8) is true, and let $\phi \in \mathcal{S}$ be arbitrary but fixed. From (35) and (36), it follows that

$$
\begin{aligned}
& \left|\frac{1}{\pi} \int_{-\pi}^{\pi} \hat{h}_{T}\left(\omega_{1}\right) \hat{\phi}\left(-\omega_{1}\right) \frac{\sin \left(N\left(\omega-\omega_{1}\right)\right)}{\omega-\omega_{1}} \mathrm{~d} \omega_{1}\right| \\
& \leq C_{10}\left(\hat{h}_{T}, \phi\right) \\
& \quad+|\hat{\phi}(-\omega)|\left|\frac{1}{\pi} \int_{-\pi}^{\pi} \hat{h}_{T}\left(\omega_{1}\right) \frac{\sin \left(N\left(\omega-\omega_{1}\right)\right)}{\omega-\omega_{1}} \mathrm{~d} \omega_{1}\right| \\
& \leq C_{10}\left(\hat{h}_{T}, \phi\right)+\|\hat{\phi}\|_{\infty} C_{1}
\end{aligned}
$$

for all $\omega \in[-\pi, \pi]$. Taking the maximum on both sides yields

$$
\begin{aligned}
& \max _{\omega \in[-\pi, \pi]} \mid \frac{1}{\pi} \int_{-\pi}^{\pi} \hat{h}_{T}\left(\omega_{1}\right) \hat{\phi}\left(-\omega_{1}\right) \frac{\sin \left(N\left(\omega-\omega_{1}\right)\right)}{\omega-\omega_{1}} \mathrm{~d} \omega_{1} \mid \\
& \leq C_{10}\left(\hat{h}_{T}, \phi\right)+\|\hat{\phi}\|_{\infty} C_{1}<\infty
\end{aligned}
$$

which completes the proof.

\section{Proof of Theorem 7}

We have

$$
\begin{aligned}
\left|\int_{-N}^{N} h_{H}(\tau) \mathrm{e}^{i \omega \tau} \mathrm{d} \tau\right| & \left|2 i \int_{0}^{N} \frac{\sin ^{2}\left(\frac{\pi}{2} \tau\right)}{\frac{\pi}{2} \tau} \sin (\omega \tau) \mathrm{d} \tau\right| \\
= & \left|\frac{2}{\pi} \int_{0}^{N} \frac{1-\cos (\pi \tau)}{\tau} \sin (\omega \tau) \mathrm{d} \tau\right| \\
\leq & \left|\frac{2}{\pi} \int_{0}^{N} \frac{\sin (\omega \tau)}{\tau} \mathrm{d} \tau\right|+\left|\frac{1}{\pi} \int_{0}^{N} \frac{\sin ((\pi-\omega) \tau)}{\tau} \mathrm{d} \tau\right| \\
& +\left|\frac{1}{\pi} \int_{0}^{N} \frac{\sin ((\pi+\omega) \tau)}{\tau} \mathrm{d} \tau\right| \\
\leq & \left|\frac{2}{\pi} \int_{0}^{\omega N} \frac{\sin (\tau)}{\tau} \mathrm{d} \tau\right|+\left|\frac{1}{\pi} \int_{0}^{(\pi-\omega) N} \frac{\sin (\tau)}{\tau} \mathrm{d} \tau\right| \\
& +\left|\frac{1}{\pi} \int_{0}^{(\pi+\omega) N} \frac{\sin (\tau)}{\tau} \mathrm{d} \tau\right| \\
< & C_{11} \mid
\end{aligned}
$$

for all $N \in \mathbb{N}$ and all $\omega \in[-\pi, \pi]$, because $\int_{0}^{x} \sin (\tau) / \tau \mathrm{d} \tau<$ $\pi$, independently of $x \in \mathbb{R}$. Using (20) and Theorem 4 gives $\lim _{N \rightarrow \infty}\left\|H f-B_{N}^{H} f\right\|_{\infty}=0$.

Similar to Theorem 1 it can be shown that $\limsup _{N \rightarrow \infty}\left\|H f-A_{N}^{H} f\right\|_{\infty}=\infty$ if and only if

$$
\sup _{t \in \mathbb{R}} \sup _{N \in \mathbb{N}} \max _{\omega \in[-\pi, \pi]}\left|\int_{-N}^{t+N} h_{H}(\tau) \mathrm{e}^{-i \omega \tau} \mathrm{d} \tau\right|=\infty .
$$

But choosing $t=N$ and $\omega=0$, and using

$$
\int_{0}^{2 N} h_{H}(\tau) \mathrm{d} \tau=\int_{0}^{2 N} \frac{\sin ^{2}\left(\frac{\pi}{2} \tau\right)}{\frac{\pi}{2} \tau} \mathrm{d} \tau>\frac{1}{\pi} \log (2 N),
$$

where the last inequality follows from (17), shows exactly this.

\section{REFERENCES}

[1] A. J. Jerri, "The Shannon sampling theorem-its various extensions and applications: A tutorial review," Proc. IEEE, vol. 65, no. 11, pp. 1565-1596, Nov. 1977.

[2] R. J. Marks, II, Advanced Topics in Shannon Sampling and Interpolation Theory. New York: Springer-Verlag, 1993.

[3] J. R. Higgins, Sampling Theory in Fourier and Signal Analysis-Foundations. London, U.K.: Oxford Univ. Press, 1996.

[4] R. L. Stens, "A unified approach to sampling theorems for derivatives and Hilbert transforms," Signal Process., vol. 5, pp. 139-151, 1983. 
[5] M. K. Habib, "Digital representations of operators on band-limited random signals," IEEE Trans. Inf. Theory, vol. 47, no. 1, pp. 173-177, Jan. 2001.

[6] M. Schetzen, Linear Time-Invariant Systems. New York: Wiley, 2002.

[7] B. Boulet, Fundamentals of Signals and Systems. Boston, MA: Charles River Media, 2006

[8] M. Zakai, "Band-limited functions and the sampling theorem," Inf. Control, vol. 8, no. 2, pp. 143-158, 1965.

[9] D. H. Mugler, "Convolution, differential equations, and entire functions of exponential type," Trans. Amer. Math. Soc., vol. 216, pp. 145-187, Feb. 1976

[10] H. Boche and U. J. Mönich, "Time domain representation of systems on bandlimited signals," in Proc. 2008 IEEE Information Theory Workshop (ITW), May 2008, pp. 51-55.

[11] S. Cambanis and E. Masry, "Zakai's class of bandlimited functions and processes: Its characterization and properties," SIAM J. Appl. Math., vol. 30, no. 1, pp. 10-21, 1976.

[12] I. W. Sandberg, "Causality and the impulse response scandal," IEEE Trans. Circuits Syst. I, Fundam. Theory Appl., vol. 50, no. 6, pp. 810-813, Jun. 2003

[13] I. W. Sandberg, "Bounded inputs and the representation of linear system maps," Circuits, Syst., Signal Process., vol. 24, no. 1, pp. 103-115, Feb. 2005.

[14] M. Ciampa, M. Franciosi, and M. Poletti, "A note on impulse response for continuous, linear, time-invariant, continuous-time systems," IEEE Trans. Circuits Syst. I, Reg.Papers, vol. 53, no. 1, pp. 106-113, Jan. 2006.

[15] M. Ciampa, M. Franciosi, and M. Poletti, "Continuous LTI systems defined on $L^{p}$ functions and $\mathcal{D}_{L}^{\prime} p$ distributions: Analysis by impulse response and convolution," IEEE Trans. Circuits Syst. I, Reg. Papers, vol. 55, no. 6, pp. 1711-1721, Jul. 2008.

[16] H. Boche and U. J. Mönich, "Local and global convergence behavior of non-equidistant sampling series," in Proc. IEEE Int. Conf. Acoustics, Speech, Signal Processing (ICASSP), Apr. 2009, pp. 2945-2948.

[17] A. H. Zemanian, Distribution Theory and Transform Analysis. New York: Dover, 1987.

[18] W. Rudin, Real and Complex Analysis, 3rd ed. New York: McGrawHill, 1987.

[19] K. Yosida, Functional Analysis. New York: Springer-Verlag, 1971.

[20] H. Boche and U. J. Mönich, "General behavior of sampling-based signal and system representation," in Proc. 2008 IEEE Int. Symp. Information Theory, Jul. 2008, pp. 2439-2443.

[21] H. Boche and U. J. Mönich, "Limits of signal processing performance under thresholding," Signal Process., vol. 89, no. 8, pp. 1634-1646, Aug. 2009.

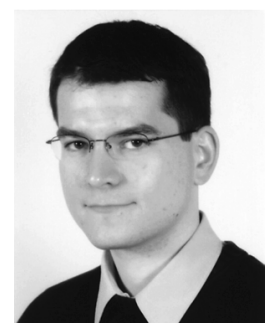

Ullrich J. Mönich (S'06) received the Dipl.-Ing. degree in electrical engineering from the Technische Universität Berlin, Germany, in 2005, where he is currently working towards the Ph.D. degree.

During winter term 2003, he was a Visiting Researcher at the University of California, Santa Barbara. His research activities comprise sampling theory, ultra-wideband mobile communications, and information theory.

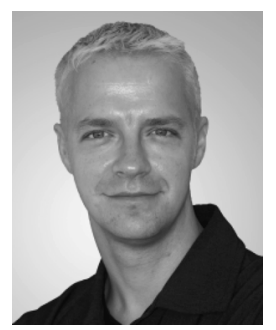

Holger Boche (M'04-SM'07) received the Dipl.-Ing. and Dr.-Ing. degrees in electrical engineering from the Technische Universität Dresden, Germany, in 1990 and 1994, respectively. He graduated in Mathematics from the Technische Universität Dresden, Germany, in 1992 and received the Dr.rer.nat. degree in pure mathematics from the Technische Universität Berlin, Germany, in 1998.

From 1994 to 1997, he did postgraduate studies in mathematics at the Friedrich-Schiller Universität Jena, Germany. In 1997, he joined the Heinrich-Hertz-Institut (HHI) für Nachrichtentechnik Berlin, Germany. Since 2002, he has been Full Professor for Mobile Communication Networks at the Technische Universität Berlin at the Institute for Communications Systems. In 2003, he became Director of the Fraunhofer German-Sino Lab for Mobile Communications, Berlin, Germany, and since 2004 he has also been Director of the Fraunhofer Institute for Telecommunications (HHI), Berlin, Germany. He was Visiting Professor at the ETH Zurich during winter term 2004 and 2006 and at KTH Stockholm during summer term 2005.

Prof. Boche received the Research Award Technische Kommunikation from the Alcatel SEL Foundation in October 2003, the Innovation Award from the Vodafone Foundation in June 2006, and the Gottfried Wilhelm Leibniz Prize from the Deutsche Forschungsgemeinschaft (German Research Foundation) in 2008. He was corecipient of the 2006 IEEE Signal Processing Society Best Paper Award and recipient of the 2007 IEEE Signal Processing Society Best Paper Award. He is a member of the IEEE Signal Processing Society SPCOM and SPTM Technical Committee. He was elected a member of the German Academy of Sciences (Leopoldina) in 2008. 